\title{
Calculation of Hilbert Borcherds Products
}

\author{
Sebastian Mayer
}

\section{CONTENTS}

1. Introduction

2. Integers in $\mathbb{Q}(\sqrt{p})$

3. Calculation of Eisenstein Series

4. The Theorem of Borcherds, Bruinier, and Bundschuh

5. Calculation of Borcherds Products

References
2000 AMS Subject Classification: Primary: 11F41

Keywords: Hilbert modular forms, Borcherds products
In [Brunier and Bundschuh 03], the authors use Borcherds lifts to obtain Hilbert modular forms. Another approach is to calculate Hilbert modular forms using the Jacquet-Langlands correspondence, which was implemented by Lassina Dembele in MAGMA. In [Mayer 09] we use [Brunier and Bundschuh 03] to determine the rings of Hilbert modular forms for $\mathbb{Q}(\sqrt{13})$ and $\mathbb{Q}(\sqrt{17})$. In the present note we give the major calculational details and present some results for $\mathcal{K}=\mathbb{Q}(\sqrt{5})$, $\mathcal{K}=\mathbb{Q}(\sqrt{13})$, and $\mathcal{K}=\mathbb{Q}(\sqrt{17})$. For calculations in the ring $\mathfrak{o}$ of integers of $\mathcal{K}$ we order $\mathfrak{o}$ by the norm of its elements and get for fixed norm, modulo multiplication by $\pm \varepsilon_{0}^{2 \mathbb{Z}}$, a finite set. We use this decomposition to describe Weyl chambers and their boundaries, to determine the Weyl vector of Borcherds products, and hence to calculate Borcherds products. As a further example we calculate Fourier expansions of Eisenstein series.

\section{INTRODUCTION}

In [Brunier and Bundschuh 03] the authors outline a path to calculate Hilbert Borcherds products for totally real number fields $\mathcal{K}$ and calculate some Borcherds products in the case $\mathcal{K}=\mathbb{Q}(\sqrt{5})$. In [Mayer 09] we use this to determine the rings of Hilbert modular forms for $\mathbb{Q}(\sqrt{13})$ and $\mathbb{Q}(\sqrt{17})$. In this note we present the major calculational details and give examples in the cases of $\mathbb{Q}(\sqrt{5})$, $\mathbb{Q}(\sqrt{13})$, and $\mathbb{Q}(\sqrt{17})$. We start with a treatment of calculations in the ring $\mathfrak{o}$ of integers of $\mathcal{K}$. Especially, we write $\mathfrak{o}$ in Lemma 2.2 as $\pm \varepsilon_{0}^{2 \mathbb{Z}} \bigcup_{m \in \mathbb{Z}} \mathcal{J}(m)$ with, for each $m \in \mathbb{Z}$, a finite set $\mathcal{J}=\mathcal{J}(m)$ of elements of norm $m$ and the fundamental unit $\varepsilon_{0}>1$.

The corresponding decomposition of $\mathfrak{o}$ is then used to describe Weyl chambers and their boundaries, to calculate Eisenstein series, and to determine the Weyl vectors of the Borcherds products. We give an algorithm to calculate the Borcherds lift of nearly holomorphic modular forms in $A_{k}^{+}\left(p, \chi_{p}\right)$. For the calculation of a basis of $A_{k}^{+}\left(p, \chi_{p}\right)$ we refer to [Brunier and Bundschuh 03] (in the case of $\mathbb{Q}(\sqrt{5})$ ) and to [Mayer 09] or [Mayer 07].

(C) A K Peters, Ltd. $1058-6458 / 2010 \$ 0.50$ per page Experimental Mathematics 19:2, page 243 


\section{INTEGERS IN $\mathbb{Q}(\sqrt{p})$}

Let $p$ be a prime number and $\mathcal{K}=\mathbb{Q}(\sqrt{p})$ the corresponding quadratic number field with ring of integers o. Denote the nontrivial field automorphism by ${ }^{-}$and for $\lambda \in \mathcal{K}$ the norm by $\mathrm{N}(\lambda)=\lambda \bar{\lambda}$ and the trace by $\mathrm{S}(\lambda)=\lambda+\bar{\lambda}$. In order to calculate Borcherds products, we have to investigate some number-theoretic properties of $\mathfrak{o}$. In particular, we give in Lemma 2.2, for fixed norm, a finite set of representatives of $\mathfrak{o}$ modulo multiplication by $\pm \varepsilon_{0}^{2}$ and use this to investigate the sets $S(m)$, which bound the union of all Weyl chambers. This is important in the calculation of Borcherds products and in addition, proves that Weyl chambers are open sets.

Furthermore, Lemma 2.2 is quite useful in calculating Fourier expansions, especially in the case of Eisenstein series.

Lemma 2.1. (Fundamental unit.) We write $\varepsilon_{0}:=x_{0}+$ $y_{0} \sqrt{p}$ for the fundamental unit of $\mathfrak{o}$ with $x_{0}, y_{0} \in \mathbb{Q}$. Then $x_{0}>0$ and $y_{0}>0$.

Proof. We have $\mathrm{N}\left(\varepsilon_{0}\right)=\varepsilon_{0} \overline{\varepsilon_{0}}= \pm 1$ and $\varepsilon_{0}=x_{0}+y_{0} \sqrt{p}$ $>1$. So we obtain $\varepsilon_{0}>1>\left|\overline{\varepsilon_{0}}\right|>0$ and conclude that $y_{0}=\left(\varepsilon_{0}-\overline{\varepsilon_{0}}\right) /(2 \sqrt{p})>0$ and $x_{0}=\left(\varepsilon_{0}+\overline{\varepsilon_{0}}\right) / 2>0$, independent of the sign of $\overline{\varepsilon_{0}}$.

This result is important in the proof of the following lemma.

Lemma 2.2. (Numbers of fixed norm.) Let $p$ be a prime number, $\mathcal{K}=\mathbb{Q}(\sqrt{p})$, and let $\mathfrak{o}$ be the ring of integers in $\mathcal{K}$. For every $m$ in $\mathbb{Z} \backslash\{0\}$ there is a finite set $\mathcal{J}$ such that

$$
\begin{aligned}
\mathcal{I} & :=\left\{\lambda \in \frac{\mathfrak{o}}{\sqrt{p}}: \mathrm{N}(\lambda)=-\frac{m}{p}\right\} \\
& =\left\{ \pm \lambda \varepsilon_{0}^{2 k}: k \in \mathbb{Z}, \lambda \in \mathcal{J}\right\} .
\end{aligned}
$$

More precisely, if we write $\breve{\lambda}_{1}+\breve{\lambda}_{2} \sqrt{p} / p:=\varepsilon_{0}^{-2} \lambda$ for all $\lambda \in \mathfrak{o} / \sqrt{p}$, we obtain that

$$
\begin{aligned}
& \mathcal{J}:=\left\{\lambda=\lambda_{1}+\lambda_{2} \sqrt{p} / p \in \frac{\mathfrak{o}}{\sqrt{p}}:\right. \\
&\left.\mathrm{N}(\lambda)=-\frac{m}{p}, \lambda_{1}>0, \lambda_{2}>0, \breve{\lambda}_{1} \breve{\lambda}_{2} \leq 0\right\}
\end{aligned}
$$

is a set of representatives of $\mathcal{I} / \sim$ with respect to the equivalence relation $\sim$ induced by multiplication by $\varepsilon_{0}^{2}$ and -1 .

For $\lambda=\lambda_{1}+\lambda_{2} \sqrt{p} / p$ in $\mathcal{J}$ we have, depending on $m=-p \mathrm{~N}(\lambda)$ and $p$, the results given in Table 1.
Proof. A detailed proof can be found in [Mayer 07, Lemma 3.2.2]. Clearly, along with $\lambda \in \mathcal{I}$, we also have $-\lambda \in \mathcal{I}$ as well as $\varepsilon_{0}^{2 k} \lambda \in \mathcal{I}$ for all $k \in \mathbb{Z}$.

For $m \in \mathbb{Z} \backslash\{0\}$ and $\lambda=\lambda_{1}+\lambda_{2} \frac{\sqrt{p}}{p} \in \mathcal{I}$ we obtain

$$
\begin{aligned}
\varepsilon_{0}^{ \pm 2} \lambda= & \lambda_{1}\left(x_{0}^{2}+p y_{0}^{2}\right) \pm \lambda_{2}\left(2 x_{0} y_{0}\right) \\
& +\sqrt{p}\left( \pm \lambda_{1}\left(2 x_{0} y_{0}\right)+\lambda_{2} \frac{x_{0}^{2}+p y_{0}^{2}}{p}\right)
\end{aligned}
$$

and $N(\lambda)=-m / p<0$; hence $p \lambda_{1}^{2}+m=\lambda_{2}^{2}$.

Given $m>0$, we let $\lambda_{2} \geq 0$ without loss of generality. From $p \lambda_{1}^{2}+m=\lambda_{2}^{2}$ it follows that $\lambda_{2}>\sqrt{p}\left|\lambda_{1}\right| \geq 0$. Calculations prove that for $\varepsilon_{0}^{2} \lambda=\tilde{\lambda}=\tilde{\lambda}_{1}+\tilde{\lambda}_{2} \frac{\sqrt{p}}{p}$, we have $\tilde{\lambda}_{2}>0$ and with $(2-1)$, that $\tilde{\lambda}_{1}>\lambda_{1} \varepsilon_{0}^{-2}$.

Since $\varepsilon_{0}^{-2}<1$ and for $\lambda \in \mathfrak{o}$ the coefficients $\lambda_{1}$ and $\lambda_{2}$ take values in a discrete set, there is $k \in \mathbb{N}$ such that $\varepsilon_{0}^{2 k} \lambda=\dot{\lambda}_{1}+\dot{\lambda}_{2} \frac{\sqrt{p}}{p}$ satisfies $\hat{\lambda}_{1}>0$ and $\dot{\lambda}_{2}>0$. For $\dot{\lambda}_{0}^{-2}=\breve{\lambda}_{1}+\breve{\lambda}_{2} \frac{\sqrt{p}}{p}$ we get $\breve{\lambda}_{2}>0$ and $\breve{\lambda}_{1}<\hat{\lambda}_{1}$ from (2-1), and the given restrictions in the lemma for $\breve{\lambda}_{2} \leq 0$ can be calculated.

The case $m<0$ can be treated analogously. Without loss of generality we then have $\lambda_{1} \geq 0$ and achieve $\bar{\lambda}_{2}^{\prime}>0$ by multiplication by some appropriate power of $\varepsilon_{0}^{2}$. Then we multiply by $\varepsilon_{0}^{-2}$ to obtain $\breve{\lambda}=\varepsilon_{0}^{-2} \hat{\lambda}$ and read the stated shape of $\mathcal{J}$ from the equation $\breve{\lambda}_{2}=0$.

Definition 2.3. We denote the complex upper half-plane by $\mathbb{H}$, and the Hilbert modular group by $\Gamma$ and its subgroup fixing $\infty=(\infty, \infty)$ by $\Gamma_{\infty}$ :

$$
T(m):=\cup_{a, b, \lambda M(a, b, \lambda)},
$$

with

$M(a, b, \lambda)=\left\{\left(\tau_{1}, \tau_{2}\right) \in \mathbb{H}^{2} ; \quad a \tau_{1} \tau_{2}+\lambda \tau_{1}+\bar{\lambda} \tau_{2}+b=0\right\}$,

where the summation is over

$$
\left\{a, b, \lambda \in \mathfrak{L}^{\prime}=\mathbb{Z}^{2} \times \frac{1}{\sqrt{p}} \mathfrak{o}: a b-\mathrm{N}(\lambda)=\frac{m}{p}\right\},
$$

and

$$
S(m):=\bigcup_{\substack{\lambda \in \mathfrak{o} / \sqrt{p} \\-\mathrm{N}(\lambda)=m / p}} M(\lambda),
$$

where

$$
M(\lambda):=\left\{\left(\tau_{1}, \tau_{2}\right) \in \mathbb{H} \times \mathbb{H}: \lambda \operatorname{Im}\left(\tau_{1}\right)+\bar{\lambda} \operatorname{Im}\left(\tau_{2}\right)=0\right\}
$$

and $T(m)$ is called Hirzebruch-Zagier divisor of discriminant $m$, where one assigns the multiplicity 1 to every irreducible component of $T(m)$. 


\begin{tabular}{|c|c|c|c|c|}
\hline & \multicolumn{2}{|c|}{$\boldsymbol{m}>\mathbf{0}$} & \multicolumn{2}{|c|}{$\boldsymbol{m}<\mathbf{0}$} \\
\hline $\boldsymbol{p}$ & $\lambda_{1} \leq \sqrt{\frac{m \alpha_{p}}{1-p \alpha_{p}}}$ & $\lambda_{2} \leq \sqrt{\frac{m}{1-p \alpha_{p}}}$ & $\lambda_{1} \leq \sqrt{\frac{-m}{p\left(1-p \alpha_{p}\right)}}$ & $\lambda_{2} \leq \sqrt{\frac{-m p \alpha_{p}}{1-p \alpha_{p}}}$ \\
\hline $\boldsymbol{p}=\mathbf{5}$ & $\lambda_{1} \leq \frac{1}{2} \sqrt{m}$ & $\lambda_{2} \leq \frac{3}{2} \sqrt{m}$ & $\lambda_{1} \leq \frac{3 \sqrt{5}}{10} \sqrt{-m}$ & $\lambda_{2} \leq \frac{\sqrt{5}}{2} \sqrt{-m}$ \\
$\boldsymbol{p}=\mathbf{1 3}$ & $\lambda_{1} \leq \frac{3}{2} \sqrt{m}$ & $\lambda_{2} \leq \frac{11}{2} \sqrt{m}$ & $\lambda_{1} \leq \frac{11}{2 \sqrt{13}} \sqrt{-m}$ & $\lambda_{2} \leq \frac{3 \sqrt{13}}{2} \sqrt{-m}$ \\
$\boldsymbol{p}=\mathbf{1 7}$ & $\lambda_{1} \leq 8 \sqrt{m}$ & $\lambda_{2} \leq 33 \sqrt{m}$ & $\lambda_{1} \leq \frac{33 \sqrt{17}}{17} \sqrt{-m}$ & $\lambda_{2} \leq 8 \sqrt{17} \sqrt{-m}$ \\
\hline
\end{tabular}

TABLE 1. Estimates for $\lambda_{1}+\lambda_{2} \sqrt{p} / p$ in the set $\mathcal{J}$ of representatives of $\mathcal{I} / \sim$, where $\alpha_{p}=\left(2 x_{0} y_{0}\right)^{2} /\left(x_{0}^{2}+p y_{0}^{2}\right)^{2}$ with the fundamental unit $\varepsilon_{0}=x_{0}+y_{0} \sqrt{p}$.

\begin{tabular}{|c||c|c|c|c|c|c|c|c|c|c|c|c|c|c|c|c|}
\hline $\boldsymbol{m}$ & $\mathbf{1}$ & $\mathbf{2}$ & $\mathbf{3}$ & $\mathbf{4}$ & $\mathbf{5}$ & $\mathbf{6}$ & $\mathbf{7}$ & $\mathbf{8}$ & $\mathbf{9}$ & $\mathbf{1 0}$ & $\mathbf{1 1}$ & $\mathbf{1 2}$ & $\mathbf{1 3}$ & $\mathbf{1 4}$ & $\mathbf{1 5}$ & $\mathbf{1 6}$ \\
\hline$\chi_{\mathbf{5}}(\boldsymbol{m})$ & 1 & -1 & -1 & 1 & & 1 & -1 & -1 & 1 & & 1 & -1 & -1 & 1 & & 1 \\
$\chi_{\mathbf{1 3}}(\boldsymbol{m})$ & 1 & -1 & 1 & 1 & -1 & -1 & -1 & -1 & 1 & 1 & -1 & 1 & & 1 & -1 & 1 \\
$\boldsymbol{\chi}_{\mathbf{1 7}}(\boldsymbol{m})$ & 1 & 1 & -1 & 1 & -1 & -1 & -1 & 1 & 1 & -1 & -1 & -1 & 1 & -1 & 1 & 1 \\
\hline
\end{tabular}

TABLE 2. $\chi_{p}(m)$ for $m \leq 16$ and $p=5,13,17$.

All divisors of Borcherds products are HirzebruchZagier divisors and conversely (cf. Remark 4.3). In [van der Geer 88], the author describes for discriminant $D$ the shape of some special sets of quadratic equations. A special case of this gives us the number of generating equations of $T(m)$ for given $p$ and $m$.

\section{Definition 2.4. (Nearly holomorphic modular forms.)}

We denote by $A_{k}\left(p, \chi_{p}\right)$ the space of nearly holomorphic modular forms of weight $k$ for the group $\Gamma_{0}(p)$ with the Dirichlet character $\chi_{p}$ given by the Legendre symbol (cf. Table 2). Here "nearly holomorphic" means that the modular forms are holomorphic on $\mathbb{H}$ and meromorphic at the cusps. Then the plus space $A_{k}^{+}\left(p, \chi_{p}\right)$ is the subspace of $A_{k}\left(p, \chi_{p}\right)$ where for $f \in A_{k}^{+}\left(p, \chi_{p}\right)$ with $f(z)=\sum_{n \in \mathbb{Z}} a(n) q^{n}, q=e^{2 \pi i z}$, we have $a(n)=0$ for all $n \in \mathbb{Z}$ with $\chi_{p}(n)=-1$.

Definition 2.5. (Weyl chamber.) For $f=\sum_{n \in \mathbb{Z}} a(n) q^{n}$ $\in A_{0}^{+}\left(p, \chi_{p}\right)$ we call $W \subset \mathbb{H} \times \mathbb{H}$ a Weyl chamber attached to $f$ if $W$ is a connected component of

$$
\mathbb{H} \times \mathbb{H} \backslash \bigcup_{\substack{n<0 \\ a(n) \neq 0}} S(-n) .
$$

Definition 2.6. $((\boldsymbol{W}, \boldsymbol{\lambda})>\mathbf{0}$.) For $W \subset \mathbb{H} \times \mathbb{H}$, especially if $W$ is a Weyl chamber, and $\lambda \in \mathfrak{o} / \sqrt{p}$ we write $(W, \lambda)=(\lambda, W)>0$ if $\lambda \operatorname{Im}\left(\tau_{1}\right)+\bar{\lambda} \operatorname{Im}\left(\tau_{2}\right)>0$ holds for all $\left(\tau_{1}, \tau_{2}\right)$ in $W$.

Lemma 2.7. (Shape of $\boldsymbol{S}(\boldsymbol{m})$.) For every prime number $p$ and every $m>0$ the set $S(m)$ is the intersection of $\mathbb{H}^{2}$ with an empty or an infinite union of hyperplanes of the real vector space $\mathbb{C}^{2}$. We have

$$
\begin{aligned}
& S(m) \\
& =\bigcup_{\lambda \in \mathcal{I}}\left\{\left(z_{1}, z_{2}\right) \in \mathbb{H} \times \mathbb{H}: \lambda \operatorname{Im}\left(z_{1}\right)+\bar{\lambda} \operatorname{Im}\left(z_{2}\right)=0\right\},
\end{aligned}
$$

where $\mathcal{I}$ is as in Lemma 2.2. In particular, $S(m)$ is invariant under the stabilizer $\Gamma_{\infty}$ of infinity.

Proof. The set $S(m)$ has the given shape by Definition 2.3. Let $\mathcal{I}$ be the set of $\lambda$ in $\mathfrak{o} / \sqrt{p}$ with $-\mathrm{N}(\lambda)=m / p$ and let $\mathcal{I}$ be nonempty, e.g., let $\lambda \in \mathcal{I}$ be an element. Clearly, the set

$$
M(\lambda)=\left\{\left(\tau_{1}, \tau_{2}\right) \in \mathbb{H} \times \mathbb{H}: \lambda \operatorname{Im}\left(\tau_{1}\right)+\bar{\lambda} \operatorname{Im}\left(\tau_{2}\right)=0\right\}
$$

is mapped onto itself by real transformations $\mathbb{H}^{2} \rightarrow$ $\mathbb{H}^{2}, \tau \mapsto \tau+r, r \in \mathbb{R}^{2}$. Let $\tau$ in $M(\lambda)$ and $k \in \mathbb{Z}$. Then defining $\tau^{(k)}:=\varepsilon_{0}^{2 k} \tau=\left(\varepsilon_{0}^{2 k} \tau_{1}, \bar{\varepsilon}_{0}^{2 k} \tau_{2}\right)$, we have

$$
\varepsilon_{0}^{-2 k} \lambda \operatorname{Im}\left(\tau_{1}^{(k)}\right)+\bar{\varepsilon}_{0}^{-2 k} \bar{\lambda} \operatorname{Im}\left(\tau_{2}^{(k)}\right)=0,
$$

so $\tau^{(k)}$ is an element of $M\left(\varepsilon_{0}^{-2 k} \lambda\right)$.

The group $\Gamma_{\infty}$ is generated by real transformations and multiplication by $\varepsilon_{0}^{2 k}(k \in \mathbb{Z})$, so we have shown the invariance under $\Gamma_{\infty}$. We rewrite $M(\lambda)$ as

$$
M(\lambda)=\left\{\left(z_{1}, z_{2}\right) \in \mathbb{H} \times \mathbb{H}: \operatorname{Im}\left(z_{2}\right)=\frac{-\lambda}{\bar{\lambda}} \operatorname{Im}\left(z_{1}\right)\right\} .
$$

Since for all $k \in \mathbb{Z} \backslash\{0\}$ we have $\varepsilon_{0}^{-2 k} /{\overline{\varepsilon_{0}}}^{-2 k} \neq 1$, the sets $M(\lambda)$ and $M\left(\varepsilon_{0}^{-2 k} \lambda\right)$ do not coincide, so $\mathcal{I}$ is either empty or has an infinite number of elements.

Remark 2.8. (Calculation of $\boldsymbol{S}(\boldsymbol{m})$.) Let $m>0$. If we use both Lemma 2.7 and Lemma 2.2, we obtain a 


\begin{tabular}{|c|c|c|c|}
\hline & weight & $\mu$ & diagonal \\
\hline \multicolumn{3}{|c|}{ Fourier expansion } \\
\hline \multicolumn{3}{|c|}{ Fourier expansion on the diagonal } \\
\hline \hline$E_{2}^{H}$ & 2 & 1 & $E_{2}^{H}(\tau, \tau)=E_{4}(\tau)$ \\
\hline \multicolumn{4}{|c|}{$1+120 g\left(h+\frac{1}{h}\right)+g^{2}\left(720+600\left(h^{2}+\frac{1}{h^{2}}\right)+120\left(h^{4}+\frac{1}{h^{4}}\right)\right)+O\left(g^{3}\right)$} \\
\hline \multicolumn{4}{|c|}{$1+240 q+2160 q^{2}+6720 q^{3}+17520 q^{4}+30240 q^{5}+O\left(q^{6}\right)$} \\
\hline \hline$E_{4}^{H}$ & 4 & 1 & $E_{4}^{H}(\tau, \tau)=\left(E_{4}(\tau)\right)^{2}=E_{8}(\tau)$ \\
\hline $1+240 g\left(h+\frac{1}{h}\right)+g^{2}\left(30240+15600\left(h^{2}+\frac{1}{h^{2}}\right)+240\left(h^{4}+\frac{1}{h^{4}}\right)\right)+O\left(g^{3}\right)$ \\
\hline \multicolumn{3}{|c|}{$1+480 q+61920 q^{2}+1050240 q^{3}+7926240 q^{4}+37500480 q^{5}+O\left(q^{6}\right)$} \\
\hline \hline$E_{6}^{H}$ & 6 & 1 & $E_{6}^{H}(\tau, \tau)=\frac{42}{67}\left(E_{4}(\tau)\right)^{3}+\frac{25}{67}\left(E_{6}(\tau)^{2}\right.$ \\
\hline $1+\frac{2520}{67} g\left(h+\frac{1}{h}\right)+g^{2}\left(\frac{7877520}{67}+\frac{2583000}{67}\left(h^{2}+\frac{1}{h^{2}}\right)+\frac{2520}{67}\left(h^{4}+\frac{1}{h^{4}}\right)\right)+O\left(g^{3}\right)$ \\
\hline $1+\frac{5040}{67} q+\frac{13048560}{67} q^{2}+\frac{1125069120}{67} q^{3}+\frac{2666085120}{67} q^{4}+\frac{31019287840}{67} q^{6}+O\left(q^{5}\right)$ \\
\hline
\end{tabular}

TABLE 3. Eisenstein series in case $p=5\left(g=\exp \left(\pi i\left(\tau_{1}+\tau_{2}\right)\right)\right.$ and $\left.h=\exp \left(\pi i\left(\tau_{1}-\tau_{2}\right) / \sqrt{p}\right)\right)$.

program for the calculation of $S(m)$. We take all positive $\lambda_{2}$ in $\frac{1}{2} \mathbb{Z}$ smaller than $\sqrt{\frac{m}{1-p \alpha_{p}}}$. Then $\lambda_{1}>0$ is uniquely determined by the formula $p \lambda_{1}^{2}+m=\lambda_{2}^{2}$. We have only to check whether $\lambda_{1} \in \mathbb{Z} / 2$. Then we have calculated $S(m)$ modulo multiplication by $\varepsilon_{0}^{2}$.

Corollary 2.9. Weyl chambers are open sets.

Proof. Each Weyl chamber is given as a component of $\mathbb{H}^{2} \backslash \bigcup_{n<0, a(n) \neq 0} S(-n)$, where the $a(n)$ are the coefficients of the principal part of a nearly holomorphic modular form of weight 0 with character $\chi_{p}$ for the group $\Gamma_{0}(p)$, so this is a finite union. By Lemma 2.7 together with Lemma 2.2, the set $S(m)$ is a locally finite union of hyperplanes, where we consider $S(m) \subset \mathbb{H}^{2}$, so $S(m)$ is a closed subset of $\mathbb{H}^{2}$. Then each of component of the complement, i.e., each Weyl chamber, is open (cf. Figure 1).

Remark 2.10. Lemma 2.2 together with Lemma 2.7 shows that $S(j)$ is a countable (or empty) union of hyperplanes

$$
E_{\lambda}=\left\{\tau \in \mathbb{H}^{2}: \operatorname{Im}\left(\tau_{1}\right)=\frac{\bar{\lambda}}{\lambda} \operatorname{Im}\left(\tau_{2}\right)\right\}
$$

which is, modulo multiplication by $\varepsilon_{0}^{2}$, a finite union of hyperplanes. The sketch shows the case $S(j)=$ $\bigcup_{m \in \mathbb{Z}}\left\{E_{\varepsilon_{0}^{2 m} a} \cup E_{\varepsilon_{0}^{2 m} b}\right\}$ in a projection of $S(j)$ and its hyperplanes and the Weyl chambers onto the imaginary parts. Each Weyl chamber is the product of its projection on the imaginary part and $\mathbb{R}^{2}$, if we write $\mathbb{H}^{2}=\mathbb{R}^{+} \times \mathbb{R}^{+} \times \mathbb{R}^{2}$.

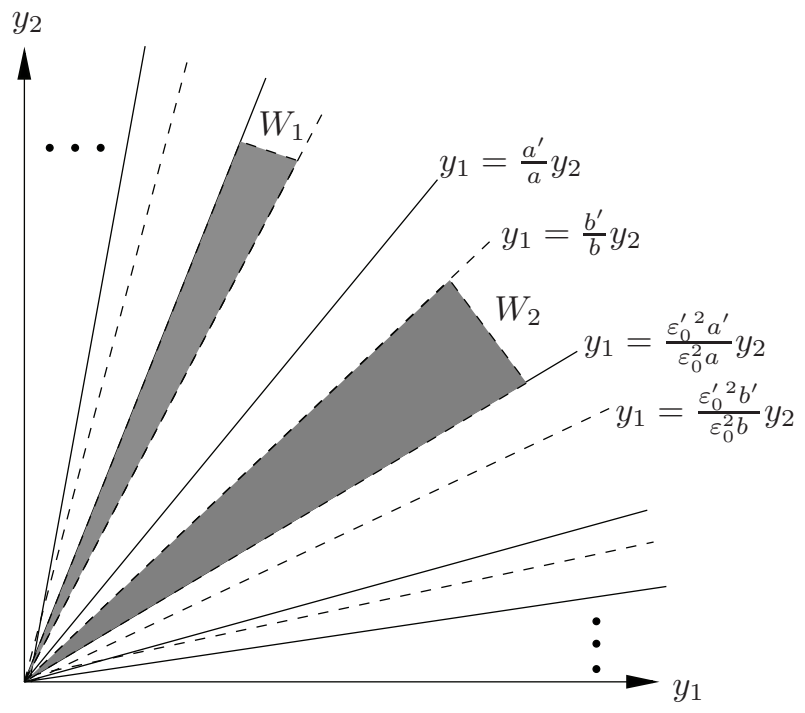

FIGURE 1. Imaginary parts of Weyl chambers.

Remark 2.11. If $W$ is a Weyl chamber attached to $f=$ $\sum_{n \in \mathbb{Z}} a(n) q^{n} \in A_{0}^{+}\left(p, \chi_{p}\right)$ and $\lambda \in \mathfrak{o} / \sqrt{p}$, then for every $a(-p \mathrm{~N}(\lambda)) \neq 0$, the condition $(\lambda, W)>0$ is equivalent to the existence of a point $\left(\tau_{1}, \tau_{2}\right) \in W$ with $\lambda \operatorname{Im}\left(\tau_{1}\right)+$ $\bar{\lambda} \operatorname{Im}\left(\tau_{2}\right)>0$.

Proof. This follows from Corollary 2.9, since all zeros of $\lambda \operatorname{Im}\left(\tau_{1}\right)+\bar{\lambda} \operatorname{Im}\left(\tau_{2}\right)$ are contained in $S(-p \mathrm{~N}(\lambda))$.

\section{CALCULATION OF EISENSTEIN SERIES}

We denote the Hilbert modular group by $\Gamma=\operatorname{SL}(2, \mathfrak{o})$ and the subgroup fixing $\infty=(\infty, \infty)$ by $\Gamma_{\infty}$. For $c, d \in \mathfrak{o}$ 


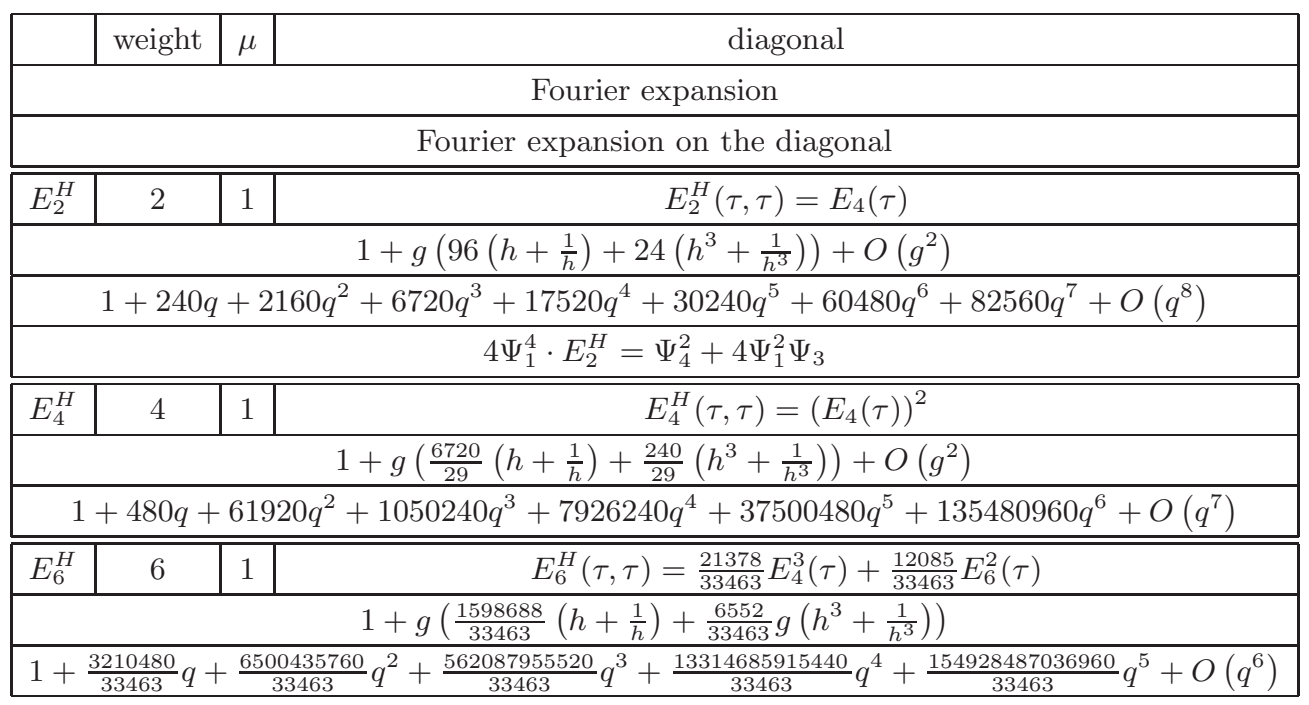

TABLE 4. Eisenstein series in case $p=13\left(g=\exp \left(\pi i\left(\tau_{1}+\tau_{2}\right)\right)\right.$ and $\left.h=\exp \left(\pi i\left(\tau_{1}-\tau_{2}\right) / \sqrt{p}\right)\right)$.

\begin{tabular}{|c|c|c|c|}
\hline & weight & $\mu$ & diagonal \\
\hline \multicolumn{4}{|c|}{ Fourier expansion } \\
\hline \multicolumn{4}{|c|}{ Fourier expansion on the diagonal } \\
\hline$E_{2}^{H}$ & 2 & 1 & $E_{2}^{H}(\tau, \tau)=E_{4}(\tau)$ \\
\hline \multicolumn{4}{|c|}{$1+g\left(84\left(h+\frac{1}{h}\right)+36\left(h^{3}+\frac{1}{h^{3}}\right)\right)+O\left(g^{2}\right)$} \\
\hline \multicolumn{4}{|c|}{$1+240 q+2160 q^{2}+6720 q^{3}+17520 q^{4}+30240 q^{5}+66312 q^{6}+82560 q^{7}+O\left(q^{8}\right)$} \\
\hline \multicolumn{4}{|c|}{$3 \Psi_{1}^{3} \cdot E_{2}^{H}=3 \Psi_{1} \cdot \Psi_{2}^{2}-\Psi_{9}$} \\
\hline$\overline{E_{4}^{H}}$ & 4 & 1 & $E_{4}^{H}(\tau, \tau)=\left(E_{4}(\tau)\right)^{2}=E_{8}(\tau)$ \\
\hline \multicolumn{4}{|c|}{$1+g\left(\frac{8760}{41}\left(h+\frac{1}{h}\right)+\frac{1080}{41}\left(h^{3}+\frac{1}{h^{3}}\right)\right)+O\left(g^{2}\right)$} \\
\hline \multicolumn{4}{|c|}{$1+480 q+61920 q^{2}+1050240 q^{3}+7926240 q^{4}+O\left(q^{5}\right)$} \\
\hline$E_{6}^{H}$ & 6 & 1 & $E_{6}^{H}(\tau, \tau)=\frac{3696}{5791} E_{4}^{3}(\tau)+\frac{2095}{5791} E_{6}^{2}(\tau)$ \\
\hline \multicolumn{4}{|c|}{$1+g\left(\frac{266364}{5791}\left(h+\frac{1}{h}\right)+\frac{8316}{5791}\left(h^{3}+\frac{1}{h^{3}}\right)\right)$} \\
\hline \multicolumn{4}{|c|}{$1+\frac{549360}{5791} q+\frac{1125094320}{5791} q^{2}+\frac{97271576640}{5791} q^{3}+\frac{2304206236080}{5791} q^{4}+O\left(q^{5}\right)$} \\
\hline$\frac{5791}{2095}\left(E_{6}^{H}-\frac{3696}{5791}\left(E_{2}^{H}\right)^{3}\right)$ & 6 & 1 & $\frac{5791}{2095}\left(E_{6}^{H}-\frac{3696}{5791}\left(E_{2}^{H}\right)^{3}\right)(\tau, \tau)=E_{6}^{2}(\tau)$ \\
\hline \multicolumn{4}{|c|}{$1-g\left(\frac{665028}{2095}\left(h+\frac{1}{h}\right)+\frac{390852}{2095}\left(h^{3}+\frac{1}{h^{3}}\right)\right)+O\left(g^{2}\right)$} \\
\hline \multicolumn{4}{|c|}{$1-1008 q+220752 q^{2}+16519104 q^{3}+399517776 q^{4}+4624512480 q^{5}+O\left(q^{6}\right)$} \\
\hline$\frac{41}{2^{4} \cdot 3^{2}}\left(E_{4}^{H}-\left(E_{2}^{H}\right)^{2}\right)$ & 4 & 1 & 0 \\
\hline \multicolumn{4}{|c|}{$13 g\left(h+\frac{1}{h}-h^{3}-\frac{1}{h^{3}}\right)+g^{2}\left(-784+349\left(h^{2}+\frac{1}{h^{2}}\right)+14\left(h^{4}+\frac{1}{h^{4}}\right)+O\left(h^{6}+\frac{1}{h^{6}}\right)\right)$} \\
\hline
\end{tabular}

TABLE 5. Eisenstein series in case $p=17\left(g=\exp \left(\pi i\left(\tau_{1}+\tau_{2}\right)\right)\right.$ and $\left.h=\exp \left(\pi i\left(\tau_{1}-\tau_{2}\right) / \sqrt{p}\right)\right)$.

\begin{tabular}{|c|c|c|c|c|c|c|c|c|c|c|c|c|c|}
\hline $\boldsymbol{p}$ & $\boldsymbol{B ( \mathbf { 1 } )}$ & $\boldsymbol{B ( 2 )}$ & $\boldsymbol{B ( 3 )}$ & $\boldsymbol{B ( 4 )}$ & $\boldsymbol{B ( 5 )}$ & $\boldsymbol{B}(\mathbf{6})$ & $\boldsymbol{B}(\mathbf{7})$ & $\boldsymbol{B}(\mathbf{8})$ & $\boldsymbol{B}(\mathbf{9})$ & $\boldsymbol{B}(\mathbf{1 0})$ & $\boldsymbol{B}(\mathbf{1 1})$ & $\boldsymbol{B}(\mathbf{1 2})$ & $\boldsymbol{B ( 1 3 )}$ \\
\hline 5 & -10 & & & -30 & -30 & -20 & & & -70 & -20 & -120 & & \\
13 & -2 & & -8 & -6 & & & & & -26 & -8 & & -24 & -14 \\
17 & -1 & -3 & & -7 & & & & -15 & -7 & & & & -14 \\
\hline
\end{tabular}

TABLE 6. Fourier coefficients of $E_{2}^{+}=1+\sum_{n \in \mathbb{N}} B(n) q^{n}$. 


\begin{tabular}{|c|c|c|c|c|c|c|c|c|c|c|c|c|c|}
\hline$p$ & $\Psi_{1}$ & $\Psi_{2}$ & $\Psi_{3}$ & $\Psi_{4}$ & $\Psi_{5}$ & $\Psi_{6}$ & $\Psi_{7}$ & $\Psi_{8}$ & $\Psi_{9}$ & $\Psi_{10}$ & $\Psi_{11}$ & $\Psi_{12}$ & $\Psi_{13}$ \\
\hline 5 & 5 & & & 15 & 15 & 10 & & & 35 & 10 & 60 & & \\
\hline 13 & 1 & & 4 & 3 & & & & & 13 & 4 & & 12 & 7 \\
\hline 17 & $1 / 2$ & $3 / 2$ & & $7 / 2$ & & & & $15 / 2$ & $7 / 2$ & & & & 7 \\
\hline$p$ & $\Psi_{14}$ & $\Psi_{15}$ & $\Psi_{16}$ & $\Psi_{17}$ & $\Psi_{18}$ & $\Psi_{19}$ & $\Psi_{20}$ & $\Psi_{21}$ & $\Psi_{22}$ & $\Psi_{23}$ & $\Psi_{24}$ & $\Psi_{25}$ & $\Psi_{26}$ \\
\hline 5 & 30 & 20 & 55 & & & 100 & 45 & 60 & & & 50 & 65 & 60 \\
\hline 13 & 6 & & 11 & 18 & & & & & 10 & 24 & & 21 & 6 \\
\hline 17 & & 4 & $31 / 2$ & $9 / 2$ & $21 / 2$ & 10 & & 6 & & & & $21 / 2$ & 21 \\
\hline$p$ & $\Psi_{27}$ & $\Psi_{28}$ & $\Psi_{29}$ & $\Psi_{30}$ & $\Psi_{31}$ & $\Psi_{32}$ & $\Psi_{33}$ & $\Psi_{34}$ & $\Psi_{35}$ & $\Psi_{36}$ & $\Psi_{37}$ & $\Psi_{38}$ & $\overline{\Psi_{39}}$ \\
\hline 5 & & & 150 & 30 & 160 & & & 80 & 60 & 105 & & & 120 \\
\hline 13 & 40 & & 30 & 16 & & & & & 24 & 39 & & 18 & 28 \\
\hline 17 & & & & 12 & & $63 / 2$ & 10 & $27 / 2$ & 12 & $49 / 2$ & & 30 & \\
\hline
\end{tabular}

TABLE 7. The weights of Borcherds products and of some of their holomorphic quotients.

\begin{tabular}{|c|c|c|c|}
\hline $\boldsymbol{p}$ & $\mathbf{5}$ & $\mathbf{1 3}$ & $\mathbf{1 7}$ \\
\hline $\boldsymbol{T}_{\mathbf{1}}$ & $\Gamma M\left(0,0, \frac{1}{5} \sqrt{5}\right)$ & $\Gamma M\left(0,0, \frac{1}{13} \sqrt{13}\right)$ & $\Gamma M\left(0,0, \frac{1}{17} \sqrt{17}\right)$ \\
$\boldsymbol{T}_{\mathbf{2}}$ & 0 & 0 & $\Gamma M\left(0,0, \frac{1}{2}+\frac{5}{34} \sqrt{17}\right)$ \\
$\boldsymbol{T}_{\mathbf{3}}$ & 0 & $\Gamma M\left(0,0, \frac{-1}{2}+\frac{5}{26} \sqrt{13}\right)$ & 0 \\
$\boldsymbol{T}_{\mathbf{4}}$ & $T_{1}+\Gamma M\left(0,-1, \frac{2}{5} \sqrt{5}\right)$ & $T_{1}+\Gamma M\left(0,-1, \frac{2}{13} \sqrt{13}\right)$ & $T_{1}+\Gamma M\left(0,0, \frac{-3}{2}+\frac{13}{34} \sqrt{17}\right)$ \\
$\boldsymbol{T}_{\mathbf{5}}$ & $\Gamma M\left(0,0, \frac{1}{2}+\frac{1}{2} \sqrt{5}\right)$ & 0 & 0 \\
$\boldsymbol{T}_{\mathbf{6}}$ & $\Gamma M\left(1,-1, \frac{-1}{2}+\frac{7}{10} \sqrt{5}\right)$ & 0 & 0 \\
$\boldsymbol{T}_{\mathbf{7}}$ & 0 & 0 & 0 \\
$\boldsymbol{T}_{\mathbf{8}}$ & 0 & 0 & 0 \\
$\boldsymbol{T}_{\mathbf{9}}$ & $T_{1}+\Gamma M\left(0,1, \frac{3}{5} \sqrt{5}\right)$ & $T_{1}+\Gamma M\left(0,0, \frac{-1}{2}+\frac{7}{26} \sqrt{13}\right)$ & $T_{1}+\Gamma M\left(0,1, \frac{3}{17} \sqrt{17}\right)$ \\
$\boldsymbol{T}_{\mathbf{1 0}}$ & $\Gamma M\left(1,1, \frac{1}{2}+\frac{1}{2} \sqrt{5}\right)$ & $\Gamma M\left(-1,-1, \frac{1}{2}+\frac{1}{26} \sqrt{13}\right)$ & 0 \\
$\boldsymbol{T}_{\mathbf{1 1}}$ & $\Gamma M\left(0,0, \frac{-1}{2}+\frac{7}{10} \sqrt{5}\right)$ & 0 & 0 \\
$\boldsymbol{T}_{\mathbf{1 2}}$ & 0 & $T_{3}+\Gamma M\left(0,-1,-1+\frac{5}{13} \sqrt{13}\right)$ & $\Gamma M\left(0,0, \frac{-1}{2}+\frac{7}{17}\right)$ \\
$\boldsymbol{T}_{\mathbf{1 3}}$ & 0 & $\Gamma M\left(0,0, \frac{3}{2}+\frac{1}{2} \sqrt{13}\right)$ & $\Gamma M\left(0,0,-2+\frac{9}{17} \sqrt{17}\right)$ \\
$\boldsymbol{T}_{\mathbf{1 4}}$ & $\Gamma M\left(1,-1, \frac{1}{2}+\frac{9}{10} \sqrt{5}\right)$ & $\Gamma M\left(1,-1, \frac{-1}{2}+\frac{11}{26} \sqrt{13}\right)$ & 0 \\
$\boldsymbol{T}_{\mathbf{1 5}}$ & $\Gamma M(1,-1,1+1 \sqrt{5})$ & 0 & $\Gamma M\left(1,-1,1+\frac{7}{17} \sqrt{17}\right)$ \\
$\boldsymbol{T}_{\mathbf{1 6}}$ & $T_{4}+\Gamma M\left(0,-1, \frac{4}{5} \sqrt{5}\right)$ & $T_{4}+\Gamma M\left(0,-1, \frac{4}{13} \sqrt{13}\right)$ & $T_{4}+\Gamma M\left(0,0, \frac{-1}{2}+\frac{9}{34} \sqrt{17}\right)$ \\
$\boldsymbol{T}_{\mathbf{1 7}}$ & 0 & $\Gamma M\left(0,0, \frac{-1}{2}+\frac{9}{26} \sqrt{13}\right)$ & $\Gamma M(0,0,4+\sqrt{17})$ \\
$\boldsymbol{T}_{\mathbf{1 8}}$ & 0 & 0 & $T_{2}+\Gamma M\left(0,1, \frac{-3}{2}+\frac{15}{34} \sqrt{17}\right)$ \\
$\boldsymbol{T}_{\mathbf{1 9}}$ & $\Gamma M\left(0,0, \frac{1}{2}+\frac{9}{10} \sqrt{5}\right)$ & $\Gamma M\left(1,1, \frac{1}{2}+\frac{7}{26} \sqrt{13}\right)$ & 0 \\
$\boldsymbol{T}_{\mathbf{2 0}}$ & $T_{5}+\Gamma M(0,-1,1+1 \sqrt{5})$ & 0 & $\Gamma M\left(0,0,-1+\frac{6}{17} \sqrt{17}\right)$ \\
$\boldsymbol{T}_{\mathbf{2 1}}$ & $\Gamma M\left(1,1, \frac{4}{5} \sqrt{5}\right)$ & $\left.0,-1+\frac{6}{13} \sqrt{13}\right)$ & 0 \\
$\boldsymbol{T}_{\mathbf{2 2}}$ & 0 & 0 & 0 \\
$\boldsymbol{T}_{\mathbf{2 3}}$ & $\boldsymbol{T}_{\mathbf{2 4}}$ & $T_{6}+\Gamma M\left(1,-1, \frac{-1}{2}+\frac{11}{10} \sqrt{5}\right)$ & $0\left(1,-1, \frac{-1}{2}+\frac{13}{34} \sqrt{17}\right)$ \\
\hline
\end{tabular}

TABLE 8. Divisors of the Borcherds products $(\Gamma=\mathrm{SL}(2, \mathfrak{o}))$.

and $\tau \in \mathbb{H}^{2}$ we extend the notion of the norm to

$$
N(c \tau+d)=\left(c \tau_{1}+d\right)\left(\bar{c} \tau_{2}+\bar{d}\right)
$$

The matrix $M=\left(\begin{array}{ll}a & b \\ c & d\end{array}\right) \in \Gamma$ operates on $\mathbb{H}^{2}$ by

$$
M \tau:=\left(\frac{a \tau_{1}+b}{c \tau_{1}+d}, \frac{\bar{a} \tau_{2}+\bar{b}}{\bar{c} \tau_{2}+\bar{d}}\right) .
$$

Definition 3.1. A Hilbert modular form $f$ of (parallel) weight $k \in \mathbb{Q}$ with multiplier system $\mu: \Gamma \rightarrow \mathbb{C} \backslash\{0\}$ for the quadratic number field $\mathcal{K}$ is a holomorphic map $f: \mathbb{H}^{2} \rightarrow \mathbb{C}$ with the property that

$$
\left.f\right|_{k} ^{\mu} M:=\mu(M)^{-1} \mathrm{~N}(c \tau+d)^{-r} f(M \tau)=f .
$$

Note that the Goetzky-Koecher principle grants that $f$ is regular at the cusps. 
For every $k \in \mathbb{N}$ the Eisenstein series of weight $2 k$ is the Hilbert modular form

$$
\begin{aligned}
E_{2 k}^{H}: \mathbb{H}^{2} & \longrightarrow \mathbb{C}, \\
\tau & \left.\longmapsto \sum_{M \in \Gamma_{\infty} \backslash \Gamma} 1\right|_{2 k} M=\sum_{M \in \Gamma_{\infty} \backslash \Gamma} \mathrm{N}(c \tau+d)^{-2 k},
\end{aligned}
$$

where $M=\left(\begin{array}{ll}a & b \\ c & d\end{array}\right)$. The Eisenstein series $E_{2 k}^{H}$ converges absolutely for $k \geq 1$ and represents a Hilbert modular form of weight $2 k$ with trivial multiplier system, which has the value 1 at the cusp $\infty$.

Siegel describes a way of calculating the Fourier coefficients of Hilbert Eisenstein series [Siegel 69]. He considers a more general definition for Hilbert Eisenstein series (he calls them Hecke Eisenstein series) than we do. We obtain

$$
\begin{aligned}
\zeta(2 k) E_{2 k}^{H}(\tau)= & F_{2 k}(\mathfrak{o}, \tau) \\
= & \zeta(2 k)+\left(\frac{(2 \pi i)^{2 k}}{(2 k-1) !}\right)^{2} \sqrt{p}^{1-4 k} \\
& \times \sum_{\substack{\nu \in \mathfrak{o}^{-1} \\
\nu \gg 0}} \sigma_{2 k-1}(\nu) e^{2 \pi i S(\nu \tau)},
\end{aligned}
$$

where

$$
\zeta(2 k)=\sum_{\text {ideals }(\mu)} \mathrm{N}\left(\mu^{-2 k}\right)=\sum_{\text {ideals }(\mu)} \mathrm{N}(\mu)^{-2 k}
$$

and

$$
\sigma_{2 k-1}(\nu)=\sum_{\substack{(t) \mid(\sqrt{p} \nu) \\ t \in \sqrt{p} \mathfrak{o}}} \mathrm{N}\left((t)^{2 k-1}\right)=\sum_{\substack{(t) \mid(\sqrt{p} \nu) \\ t \in \sqrt{p} \mathfrak{o}}} \mathrm{N}(t)^{2 k-1} .
$$

The norm $\mathrm{N}(\mu)$ of a prime ideal $(\mu)$ is given by the norm of a generating element $\mu \in \mathfrak{o}$.

Note that we have written

$$
\zeta(2 k) E_{2 k}^{H}(\tau)=\zeta(2 k)+\sum_{\nu} a_{\nu} e^{2 \pi i S(\nu \tau)}
$$

with some known $a_{\nu}$, since we can calculate the finite sum $\sigma_{2 k-1}(\nu)$ if we can calculate a set of representatives of $\mathfrak{o} / \mathfrak{o}^{*}$ ordered by the corresponding absolute value of the norm. We also need a way to order $\mathfrak{d}^{-1}=\frac{1}{\sqrt{p}} \mathfrak{o}$. Both can be achieved by Lemma 2.2.

In order to calculate $\zeta(2 k)$, Siegel advises that one restrict the Fourier expansion of $E_{2 k}^{H}$ to the diagonal Diag $=\left\{\tau \in \mathbb{H}^{2}: \tau_{1}=\tau_{2}\right\}$, which yields an elliptic modular form of weight $4 k$. A basis of all elliptic modular forms of weight $4 k$ is known, and $E_{2 k}^{H}(\tau)$ tends to 1 as the imaginary part of $\tau$ goes to infinity. Hence we can determine $\zeta(2 k)$ by linear algebra.
Remark 3.2. Some of the (truncated) Fourier expansions of Eisenstein series can be found in Tables 3, 4, and 5 .

\section{THE THEOREM OF BORCHERDS, BRUINIER, AND BUNDSCHUH}

Hilbert Borcherds products are Hilbert modular forms that vanish on some Hirzebruch-Zagier divisors $T(m)$, have absolutely convergent product expansion on Weyl chambers, and are lifts of modular forms in $A_{0}^{+}\left(p, \chi_{p}\right)$. We give the theorem of Borcherds, which is [Borcherds 98, Theorem 13.3], about Borcherds products in the version of Bruinier and Bundschuh; compare [Brunier and Bundschuh 03, Theorem 9] and [Brunier and Bundschuh 03, Theorem 3.1].

\section{Theorem 4.1. (Borcherds, Bruinier, Bundschuh.)}

Let $f=\sum_{n \in \mathbb{Z}} a(n) q^{n} \in A_{0}^{+}\left(p, \chi_{p}\right)$ and assume that $s(n) a(n) \in \mathbb{Z}$ for all $n<0$, where $s(n)=2$ if $p \mid n$ and $s(n)=1$ otherwise. Then there is a meromorphic function $\Psi$ on $\mathbb{H} \times \mathbb{H}$ with the following properties:

(i) $\Psi$ is a meromorphic modular form for $\Gamma$ with some multiplier system of finite order. The weight of $\Psi$ is equal to the constant coefficient $a(0)$ of $f$.

(ii) The divisor of $\Psi$ is determined by the principal part of $f$. It equals

$$
\sum_{n<0} s(n) a(n) T(-n)
$$

(iii) Let $W \subset \mathbb{H} \times \mathbb{H}$ be a Weyl chamber attached to $f$ and put $N=\min \{n ; a(n) \neq 0\}$. The function $\Psi$ has the Borcherds product expansion

$$
\begin{aligned}
\Psi\left(\tau_{1}, \tau_{2}\right)= & \mathbf{e}\left(\rho_{W} \tau_{1}+\overline{\rho_{W}} \tau_{2}\right) \\
& \times \prod_{\substack{\nu \in \mathfrak{o} / \sqrt{p} \\
(\nu, W)>0}}\left(1-\mathbf{e}\left(\nu \tau_{1}+\bar{\nu} \tau_{2}\right)\right)^{s(p \nu \bar{\nu}) a(p \nu \bar{\nu})},
\end{aligned}
$$

where $\mathbf{e}(\tau)=e^{2 \pi i \tau}$. Here $\rho_{W}$ and $\overline{\rho_{W}}$ are algebraic numbers in $K$ that can be computed explicitly. The product converges normally for all $\tau \in W$ with $\operatorname{Im}\left(\tau_{1}\right) \operatorname{Im}\left(\tau_{2}\right)>|N| / p$ outside the set of poles.

(iv) There is a positive integer c such that $\Psi^{c}$ has integral rational Fourier coefficients with greatest common divisor 1 .

Definition 4.2. If $W$ is a Weyl chamber and $n$ an integer, we define the set

$$
R(n):=\{\lambda \in \mathfrak{o} / \sqrt{p}: \lambda>0, \mathrm{~N}(\lambda)=n / p\}
$$




\begin{tabular}{|c|c|c|c|}
\hline$n$ & $p=5$ & $p=13$ & $p=17$ \\
\hline 1 & $\left\{\frac{1}{5} \sqrt{5}\right\}$ & $\left\{\frac{1}{13} \sqrt{13}\right\}$ & $\left\{\frac{1}{17} \sqrt{17}\right\}$ \\
\hline 2 & \{\} & \{\} & $\left\{-\frac{1}{2}+\frac{5}{34} \sqrt{17}, \frac{1}{2}+\frac{5}{34} \sqrt{17}\right\}$ \\
\hline 3 & \{\} & $\left\{-\frac{1}{2}+\frac{5}{26} \sqrt{13}, \frac{1}{2}+\frac{5}{26} \sqrt{13}\right\}$ & \{\} \\
\hline 4 & $\left\{\frac{2}{5} \sqrt{5}\right\}$ & $\left\{\frac{2}{13} \sqrt{13}\right\}$ & $\left\{-\frac{3}{2}+\frac{13}{34} \sqrt{17}, \frac{3}{2}+\frac{13}{34} \sqrt{17}, \frac{2}{17} \sqrt{17}\right\}$ \\
\hline 5 & $\left\{\frac{1}{2} \sqrt{5}-\frac{1}{2}\right\}$ & \{\} & \{\} \\
\hline 8 & \{\} & \{\} & $\left\{\begin{array}{c}\frac{1}{2}+\frac{7}{34} \sqrt{17}, 1+\frac{5}{17} \sqrt{17}, \\
-1+\frac{5}{17} \sqrt{17},-\frac{1}{2}+\frac{7}{34} \sqrt{17}\end{array}\right\}$ \\
\hline 9 & $\left\{\frac{3}{5} \sqrt{5}\right\}$ & $\left\{\begin{array}{c}\frac{1}{2}+\frac{7}{26} \sqrt{13}, \frac{3}{13} \sqrt{13} \\
-\frac{1}{2}+\frac{7}{26} \sqrt{13}\end{array}\right\}$ & $\left\{\frac{3}{17} \sqrt{17}\right\}$ \\
\hline 10 & \{\} & \{\} & \{\} \\
\hline 11 & $\left\{\begin{array}{l}\frac{1}{2}+\frac{7}{10} \sqrt{5} \\
-\frac{1}{2}+\frac{7}{10} \sqrt{5}\end{array}\right\}$ & \{\} & \{\} \\
\hline 12 & \{\} & $\left\{1+\frac{5}{13} \sqrt{13},-1+\frac{5}{13} \sqrt{13}\right\}$ & \{\} \\
\hline 13 & \{\} & $\left\{-\frac{3}{2}+\frac{1}{2} \sqrt{13}\right\}$ & $\left\{-2+\frac{9}{17} \sqrt{17}, 2+\frac{9}{17} \sqrt{17}\right\}$ \\
\hline 16 & $\left\{\frac{4}{5} \sqrt{5}\right\}$ & $\left\{\frac{4}{13} \sqrt{13}\right\}$ & $\left.\begin{array}{c}\frac{4}{17} \sqrt{17}, 3+\frac{13}{17} \sqrt{17} \\
\frac{1}{2}+\frac{9}{34} \sqrt{17},-\frac{1}{2}+\frac{9}{34} \sqrt{17} \\
-3+\frac{13}{17} \sqrt{17}\end{array}\right\}$ \\
\hline 17 & \{\} & $\left\{\frac{1}{2}+\frac{9}{26} \sqrt{13},-\frac{1}{2}+\frac{9}{26} \sqrt{13}\right\}$ & $\{\sqrt{17}-4\}$ \\
\hline 18 & \{\} & \{\} & $\left\{\frac{3}{2}+\frac{15}{34} \sqrt{17},-\frac{3}{2}+\frac{15}{34} \sqrt{17}\right\}$ \\
\hline 19 & $\left\{\begin{array}{l}\frac{1}{2}+\frac{9}{10} \sqrt{5} \\
-\frac{1}{2}+\frac{9}{10} \sqrt{5}\end{array}\right\}$ & \{\} & $\left\{-1+\frac{6}{17} \sqrt{17}, 1+\frac{6}{17} \sqrt{17}\right\}$ \\
\hline 20 & $\{-1+\sqrt{5}\}$ & \{\} & \{\} \\
\hline 23 & \{\} & $\left\{1+\frac{6}{13} \sqrt{13},-1+\frac{6}{13} \sqrt{13}\right\}$ & \{\} \\
\hline 24 & \{\} & \{\} & \{\} \\
\hline 25 & $\{\sqrt{5}\}$ & $\left\{\frac{5}{13} \sqrt{13}\right\}$ & $\left\{\frac{5}{17} \sqrt{17}\right\}$ \\
\hline
\end{tabular}

TABLE 9. $R(W,-n)$ : For $p \in\{5,13,17\}$ and $n \in\{6,7,14,15,21,22\}$ the set $R(W,-n)$ is empty.

and write $R(W, n)$ for the finite set

$$
\begin{aligned}
& R(W, n)=\left\{\lambda \in R(n): \lambda \operatorname{Im}\left(\tau_{1}\right)+\bar{\lambda} \operatorname{Im}\left(\tau_{2}\right)<0, \quad(4-1)\right. \\
&\left.\varepsilon_{0}^{2} \lambda \operatorname{Im}\left(\tau_{1}\right)+\bar{\varepsilon}_{0}^{2} \bar{\lambda} \operatorname{Im}\left(\tau_{2}\right)>0, \forall \tau \in W\right\} .
\end{aligned}
$$

From [Brunier and Bundschuh 03] we take the following remark to Theorem 4.1.

Remark 4.3. Additionally, we have

1. For all $\tau \in W$ and $y_{1}=\operatorname{Im}\left(\tau_{1}\right)$ and $y_{2}=\operatorname{Im}\left(\tau_{2}\right)$ the Weyl vector $\left(\rho_{W}, \overline{\rho_{W}}\right)$ is given by

$$
\begin{aligned}
& \rho_{W} y_{1}+\overline{\rho_{W}} y_{2} \\
& =\frac{1}{\varepsilon_{0}+\overline{\varepsilon_{0}}} \sum_{n<0} s(n) a(n) \sum_{\lambda \in R(W, n)}\left(\varepsilon_{0} \lambda y_{1}+\overline{\varepsilon_{0}} \bar{\lambda} y_{2}\right) .
\end{aligned}
$$

and

$$
\overline{\rho_{W}}=\frac{1}{\varepsilon_{0}+\overline{\varepsilon_{0}}} \sum_{n<0} s(n) a(n) \sum_{\lambda \in R(W, n)} \bar{\lambda} \overline{\varepsilon_{0}} .
$$


Proof. Since Weyl chambers are open in $\mathbb{H}^{2}$ by Corollary 2.9, equation (4-2) holds for $\tau+\left(\delta_{1}, \delta_{2}\right)$ if $\delta_{1} \geq 0$ and $\delta_{2} \geq 0$ are sufficiently small. We subtract both equations and get

$$
\begin{aligned}
& \rho_{W} \delta_{1}+\overline{\rho_{W}} \delta_{2} \\
& \quad=\frac{1}{\varepsilon_{0}+\overline{\varepsilon_{0}}} \sum_{n<0} s(n) a(n) \sum_{\lambda \in R(W, n)}\left(\varepsilon_{0} \lambda \delta_{1}+\overline{\varepsilon_{0}} \bar{\lambda} \delta_{2}\right) .
\end{aligned}
$$

Differentiation by $\delta_{1}$ and $\delta_{2}$ gives the stated result.

Lemma 4.5. (Choice of Weyl chamber.) Let $m=-n$ be a natural number and let $\tau \in \mathbb{H}^{2}$ be a point. Then $W(\tau)$ defines the Weyl chamber attached to $\tilde{\tau}:=\tau+(i \bar{\delta}, i \delta)$ for sufficiently small $\delta \in \mathcal{K}, \delta \geq 0$, in the following sense: If $\tau$ is contained in a Weyl chamber, then we define $W(\tau)$ to be this Weyl chamber $(\delta=0)$. Otherwise, if $\operatorname{Im}\left(\tau_{1}\right) \neq$ $\operatorname{Im}\left(\tau_{2}\right)$, then there are a Weyl chamber, which we denote by $W(\tau)$, and some $\delta_{0}>0$ such that for $\delta=\left(i \delta_{1}, i \delta_{1}\right)$ we have $\tau+\delta \in W(\tau)$ for all $0<\delta_{1}<\delta_{0}, \delta_{1} \in \mathbb{Q}$.

In the case that $\tau$ is not contained in a Weyl chamber and $\operatorname{Im}\left(\tau_{1}\right)=\operatorname{Im}\left(\tau_{2}\right)$, there are a unique Weyl chamber, which we denote by $W(\tau)$, and some $\delta_{0}>0$ such that $\tau+\left(-i \delta_{2} \sqrt{p}, i \delta_{2} \sqrt{p}\right)$ is contained in $W(\tau)$ for all $0<$ $\delta_{2}<\delta_{0}$ with $\delta_{2} \in \mathbb{Q}$.

Our standard choice for $\tau$ will be $\tau=\left(-i \overline{\varepsilon_{0}}+i \varepsilon_{0}\right)$ and $\tilde{\tau}:=\left(-i \overline{\varepsilon_{0}}+i \bar{\delta}, i \varepsilon_{0}+i \delta\right)$.

Proof. If $\tau \in \mathbb{H}$ is not contained in a Weyl chamber, then $\tau \in S(m)$. By Lemma 2.7 we know that $S(m)$ is a finite union of hyperplanes $M(\lambda)$ modulo multiplication by $\varepsilon_{0}^{2}$. The projection of these hyperplanes onto the imaginary parts are straight lines through 0 intersected with $\mathbb{H}^{2}$. Hence for $\tau \in S(m)$, the point $\left(\operatorname{Im}\left(\tau_{1}\right), \operatorname{Im}\left(\tau_{2}\right)\right)$ lies on the straight line through 0 with direction $\left(\operatorname{Im}\left(\tau_{1}\right), \operatorname{Im}\left(\tau_{2}\right)\right)$, and the choice of $W(\tau)$ described in the lemma is unique and well defined.

With respect to Lemma 2.11 it suffices to choose one point in $W$ in order to calculate Borcherds products on $W$ (without knowing about the concrete shape of $W$ ). Now we can easily calculate $R(W, n)$.

Lemma 4.6. (Calculation of $\boldsymbol{R}(\boldsymbol{W}, \boldsymbol{n})$.) Let $m=-n$ be a natural number and $\tau \in W$ for some Weyl chamber $W$. Then $R(W, n)$ can be calculated by the following algorithm: For every element $\lambda$ in a set of representatives of $R(-m)$ modulo multiplication by $\varepsilon_{0}^{2}$ (such as $\mathcal{J} \bigcup-\mathcal{J}$ in Lemma 2.2) do

(i) Multiply $\lambda$ by $\varepsilon_{0}^{2}$ (and denote the result again by $\lambda$ ) until $\lambda y_{1}+\bar{\lambda} y_{2}>0$ for the imaginary part $y$ of $\tau$.

\section{(ii) Multiply $\lambda$ by $\varepsilon_{0}^{-2}$ until $\lambda y_{1}+\bar{\lambda} y_{2}<0$.}

The resulting $M(\lambda)$ is an element of $R(W, n)$ and this procedure gives all of its elements when applied to all $\lambda$ in $R(-m) / \varepsilon_{0}^{2}$.

Proof. We have $\varepsilon_{0}>1$ and $\mathrm{N}\left(\varepsilon_{0}\right)= \pm 1$, so $0<\overline{\varepsilon_{0}^{2}}=$ $\varepsilon_{0}^{-2}<1$. Let $\tau \in \mathbb{H}^{2}$ and $\lambda \in \mathfrak{o} / \sqrt{p}$ with $\lambda>0$. Write $y_{1}=\operatorname{Im}\left(\tau_{1}\right)$ and $y_{2}=\operatorname{Im}\left(\tau_{2}\right)$. Then $\bar{\lambda}=\mathrm{N}(\lambda) / \lambda=$ $-\frac{m}{p} \lambda<0$ and we get

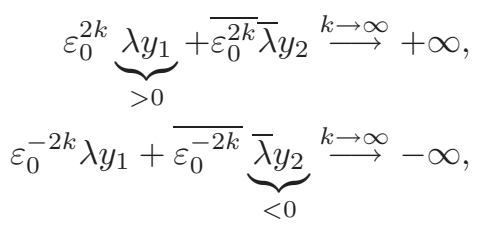

and

$$
\underbrace{\varepsilon_{0}^{2}}_{>1} \underbrace{\lambda y_{1}}_{>0}+\underbrace{\overline{\varepsilon_{0}^{2}}}_{<1} \underbrace{\bar{\lambda} y_{2}}_{<0}>\lambda y_{1}+\bar{\lambda} y_{2} .
$$

So the algorithm described in the lemma gives some $\tilde{\lambda}=$ $\varepsilon_{0}^{2 k} \lambda$ with $\tilde{\lambda} \in R(W,-n), k \in \mathbb{Z}$, and clearly it suffices to apply this algorithm to a set of representatives of $\mathcal{I}$ modulo multiplication by $\varepsilon_{0}^{2}$.

Remark 4.7. (Interpretation of $\boldsymbol{R}(\boldsymbol{W}, \boldsymbol{n})$.) From (4-1), we get that if $W$ is a Weyl chamber attached to $f_{n}$, then the boundary of $W$ in $\mathbb{H}^{2}$ is a subset of

$$
\bigcup_{\lambda \in R(W, n)}\left(M(\lambda) \bigcup M\left(\varepsilon_{0}^{2} \lambda\right)\right) .
$$

In particular, the boundary is the union of two $M(\mu)$.

\section{CALCULATION OF BORCHERDS PRODUCTS}

We investigate the remaining tasks for the concrete calculation of Borcherds products as described in Theorem 4.1. A basis of $A_{0}^{+}\left(p, \chi_{p}\right)$ is calculated via Eisenstein series in some space $M_{k}(p, 1)$, and rational functions in $\eta$ and $\eta^{(p)}$ in the cases $p \in\{5,13,17\}$ (cf. [Brunier and Bundschuh 03] in case $p=5$, [Mayer 09] in case $p=13$, and $p=17$, and [Mayer 07] in the cases $p=5, p=13$, and $p=17)$.

We will determine the multiplier system of a Borcherds product from the Weyl vector, as was suggested by Bruinier, and give a method to calculate the Fourier expansion of a Borcherds product up to arbitrary finite degree. For calculations in $A_{k}\left(p, \chi_{p}\right)$ note the following remark:

Remark 5.1. (Precision invariant under multiplication and division.) If $f$ is a meromorphic modular form 
with $f=q^{k} \sum_{n=0}^{M} a(n) q^{n}+O\left(q^{k+M+1}\right)$ and $a(0) \neq 0$, then we say that $f$ is given with precision $M+1$. Then the product and the quotient of two modular forms given with precision $N$ is given with precision $N$ again. The same holds for the inverse of a modular form. So, in order to determine the first $N$ coefficients of a product or quotient of Fourier expansions, the first $N$ coefficients have to be determined for each of the factors.

Remark 5.2. We can show by calculation of Fourier exponents that the Eisenstein series $E_{2}^{+}$considered in [Brunier and Bundschuh 03] is a theta nullwert in case $p \in\{5,13,17\}$. In particular, we set

$$
M_{5}:=\left(\begin{array}{cccc}
2 & 1 & & \\
1 & 2 & 1 & \\
& 1 & 4 & 5 \\
& & 5 & 10
\end{array}\right), \quad M_{13}:=\left(\begin{array}{cccc}
2 & 1 & & \\
1 & 4 & 3 & \\
& 3 & 10 & 13 \\
& & 13 & 26
\end{array}\right)
$$

and

$$
M_{17}:=\left(\begin{array}{cccc}
2 & 1 & & \\
1 & 4 & 1 & \\
& 1 & 10 & 17 \\
& & 17 & 34
\end{array}\right)
$$

The inverse matrices $M_{p}^{-1}$ are each contained in $(\mathbb{Z} / p)^{4 \times 4}$, so the functions

$$
z \mapsto \sum_{g \in \mathbb{Z}^{4}} e^{\pi i g^{t} M_{p} g z}
$$

are modular forms for $\Gamma_{0}(p)$. Then we can compare Fourier coefficients and obtain

$$
E_{2}^{+}(z)=\sum_{g \in \mathbb{Z}^{4}} e^{\pi i g^{t} M_{p} g z} \quad \text { for all } p \in\{5,13,17\}
$$

With [Brunier and Bundschuh 03, Theorems 6 and 9] we can use $E_{2}^{+}$to calculate the weights of the Borcherds products (cf. Tables 6 and 7 as well as Tables 8 and 9).

Next, we investigate the weights and multiplier systems possible for Hilbert modular forms following the work of [Gundlach 88]. We investigate the multiplier system of Borcherds products in dependence of the Weyl vector. In the cases $p \in\{5,13,17\}$, this suffices to determine the multiplier system of Borcherds products.

Definition 5.3. For all $w \in \mathfrak{o}$ we define

$$
T_{w}=\left(\left(\begin{array}{cc}
1 & w \\
0 & 1
\end{array}\right),\left(\begin{array}{cc}
1 & \bar{w} \\
0 & 1
\end{array}\right)\right)
$$

and write

$$
J=\left(\left(\begin{array}{cc}
0 & 1 \\
-1 & 0
\end{array}\right),\left(\begin{array}{cc}
0 & 1 \\
-1 & 0
\end{array}\right)\right)
$$

and $T:=T_{1}$.

Remark 5.4. (Symmetric multiplier systems.) Let $\mu$ be a multiplier system. We define $\bar{\mu}(M)=\mu(\bar{M})$ for all matrices $M \in \mathrm{SL}(2, \mathfrak{o})$, where $\bar{M}$ is the matrix derived from $M$ by componentwise conjugation. Then $\bar{\mu}$ is again a multiplier system and is given by $\bar{\mu}(J)=\mu(J), \bar{\mu}(T)=$ $\mu(T)$, and $\bar{\mu}\left(T_{w}\right)=\mu\left(T \cdot T_{w}^{-1}\right)=\frac{\mu(T)}{\mu\left(T_{w}\right)}$. In case $\mu=\bar{\mu}$, we call $\mu$ symmetric. In [Mayer 09] we prove the following theorem.

Theorem 5.5. (Multiplier systems of Borcherds products.) If $\Psi$ is a Borcherds product with multiplier system $\mu$, then the values $\mu\left(T_{\lambda}\right)$ of all translations $T_{\lambda}, \lambda \in \mathfrak{o}$, can be determined by $\mu\left(T_{\lambda}\right)=\mathbf{e}\left(\left(\mathrm{S}\left(\rho_{W} \lambda\right)\right)\right.$. Especially in the case $\mathbb{Q}(\sqrt{13})$ and $\mathbb{Q}(\sqrt{17})$, the multiplier system can be read from the Weyl vector.

Remark 5.6. For $\mathbb{Q}(\sqrt{5})$, only the trivial multiplier system exists.

Proof. Let $\Psi$ be a Borcherds product with multiplier system $\mu$. Then $\Psi$ has the Fourier expansion

$$
\begin{aligned}
\Psi\left(\tau_{1}, \tau_{2}\right)= & \mathbf{e}\left(\rho_{W} \tau_{1}+\overline{\rho_{W}} \tau_{2}\right) \\
& \times \prod_{\substack{\nu \in \mathfrak{o} / \sqrt{p} \\
(\nu, W)>0}}\left(1-\mathbf{e}\left(\nu \tau_{1}+\bar{\nu} \tau_{2}\right)\right)^{s(p \nu \bar{\nu}) a(p \nu \bar{\nu})},
\end{aligned}
$$

and the product is invariant with respect to the operation of $T$ and $T_{w}$, since $\mathbf{e}\left(\nu \tau_{1}+\bar{\nu} \tau_{2}\right)$ itself is invariant.

So we have $\mu(T)=\mathbf{e}\left(\mathrm{S}\left(\rho_{W}\right)\right)$ and $\mu\left(T_{w}\right)=$ $\mathbf{e}\left(\mathrm{S}\left(\rho_{W} w\right)\right)$. By [Mayer 09, Corollary 23 and 24], in case $p=13$ and $p=17$ the multiplier system $\mu$ is uniquely determined by $\mu(T)$ and $\mu\left(T_{w}\right)$. Hence in the cases $p=13$ and $p=17$, the multiplier system can be read from the Weyl vector.

Definition 5.7. If $f$ is a Hilbert modular form with Fourier expansion

$$
f(\tau)=\sum_{\substack{a, b \in \mathcal{Z} \\ a \geq 0 \\|b| \leq \sqrt{p} a}} c(a, b) g^{a} h^{b}
$$

where $g=\mathbf{e}\left(\left(\tau_{1}+\tau_{2}\right) / 2\right)$ and $h=\mathbf{e}\left(\left(\tau_{1}-\tau_{2}\right) / 2 \sqrt{p}\right)$, and there is $N \in \mathbb{N}$ such that $c(a, b)$ is known for all $a \leq N$, $|b| \leq \sqrt{p} a$, then $f$ and

$$
\sum_{\substack{a, b \in \mathcal{Z} \\ 0 \leq a \leq N \\|b| \leq \sqrt{p} a}} c(a, b) g^{a} h^{b}
$$




\begin{tabular}{|c|c|c|c|}
\hline$\overline{\Psi_{k}}$ & weight & Fourier expansion & divisor \\
\hline$\mu$ & other & Fourier expansion on the diagonal (if not 0 ) & diagonal \\
\hline$\Psi_{1}$ & 5 & $\bar{g} g\left(h-\frac{1}{h}\right)-10 g^{2}\left(h^{2}-\frac{1}{h^{2}}\right)-g^{2}\left(h^{4}-\frac{1}{h^{4}}\right)+O\left(g^{3}\right)$ & $T_{1}$ \\
\hline 1 & \multicolumn{3}{|c|}{$\bar{\Psi}_{1}=-\Psi_{1}$} \\
\hline$\frac{\Psi_{4}}{\Psi_{1}}$ & 10 & $g\left(h+\frac{1}{h}\right)+g^{2}\left(454+228\left(h^{2}+\frac{1}{h^{2}}\right)+\left(h^{4}+\frac{1}{h^{4}}\right)\right)$ & $\overline{T_{4}-T_{1}}$ \\
\hline 1 & $\left(\frac{\Psi_{4}}{\Psi_{1}}\right)=\frac{\Psi_{4}}{\Psi_{1}}$ & $2 g+912 g^{2}+101304 g^{3}-632704 g^{4}+O\left(g^{5}\right)$ & $2 E_{4}^{2} \cdot \Delta$ \\
\hline$\Psi_{4}$ & 15 & $g^{2}\left(h^{2}-\frac{1}{h^{2}}\right)+216 g^{3}\left(h+h^{3}-\frac{1}{h}-\frac{1}{h^{3}}\right)+O\left(g^{4}\right)$ & $T_{4}$ \\
\hline 1 & \multicolumn{3}{|c|}{$\bar{\Psi}_{4}=-\Psi_{4}$} \\
\hline 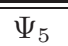 & 15 & $\overline{g^{2}-275 g^{3}\left(h+\frac{1}{h}\right)-g^{3}\left(h^{5}+\frac{1}{h^{5}}\right)+O\left(g^{4}\right)}$ & $T_{5}$ \\
\hline 1 & $\bar{\Psi}_{5}=\Psi_{5}$ & $g^{2}-552 g^{3}+8640 g^{4}+116000 g^{5}+O\left(g^{6}\right)$ & $E_{6} \cdot \Delta^{2}$ \\
\hline$\Psi_{6}$ & 10 & $1-264 g\left(h+\frac{1}{h}\right)+O\left(g^{2}\right)$ & $T_{6}$ \\
\hline 1 & $\bar{\Psi}_{6}=\Psi_{6}$ & $1-528 g-201168 g^{2}+61114944 g^{3}+O\left(g^{4}\right)$ & $E_{4}^{2} E_{6}^{2}$ \\
\hline$\overline{\Psi_{9}}$ & 35 & $g^{3}\left(h^{3}-\frac{1}{h^{3}}\right)+3555 g^{4}\left(h^{2}+h^{4}-\frac{1}{h^{2}}-\frac{1}{h^{4}}\right)+O\left(g^{5}\right)$ & $T_{9}$ \\
\hline 1 & \multicolumn{3}{|c|}{$\bar{\Psi}_{9}=-\Psi_{9}$} \\
\hline$\Psi_{10}$ & 10 & 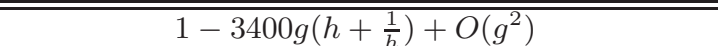 & $\overline{T_{10}}$ \\
\hline 1 & $\bar{\Psi}_{10}=\Psi_{10}$ & $1-6800 g-3061200 g^{2}-256574400 g^{3}+O\left(g^{4}\right)$ & $\frac{5^{2}}{3^{3}} E_{4}^{2} E_{6}^{2}-\frac{2 \cdot 7^{2}}{3^{3}} E_{4}^{5}$ \\
\hline$\Psi_{11}$ & 60 & $-g^{6}+3256 g^{7}\left(h+\frac{1}{h}\right)+g^{7}\left(h^{7}+\frac{1}{h^{7}}\right)+O\left(g^{8}\right)$ & $T_{11}$ \\
\hline 1 & $\bar{\Psi}_{11}=\Psi_{11}$ & $-g^{6}+6514 g^{7}+O\left(g^{8}\right)$ & \\
\hline 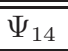 & 30 & $1+1+25704 g\left(h+\frac{1}{h}\right)+O\left(g^{2}\right)$ & $T_{14}$ \\
\hline 1 & $\bar{\Psi}_{14}=\Psi_{14}$ & $1+51408 g+146187664 g^{2}+O\left(g^{3}\right)$ & \\
\hline$\overline{\Psi_{15}}$ & 20 & $1-22425 f\left(h+\frac{1}{h}\right)+O\left(g^{2}\right)$ & $T_{15}$ \\
\hline 1 & $\bar{\Psi}_{15}=\Psi_{15}$ & $1-44850 g-428741775 g^{2}+O\left(g^{3}\right)$ & \\
\hline
\end{tabular}

TABLE 10. Borcherds products in case $p=5$ for the Weyl chamber $W\left(-i \overline{\varepsilon_{0}}, i \varepsilon_{0}\right)\left(\left(g=\exp \left(\pi i\left(\tau_{1}+\tau_{2}\right)\right)\right.\right.$ and $h=$ $\left.\left.\exp \left(\pi i\left(\tau_{1}-\tau_{2}\right) / \sqrt{p}\right)\right)\right)$.

are said to be given with precision $g^{N}$. Here $\mathcal{Z}$ is a rational ideal in $\mathbb{Q}$ depending on the multiplier system of $f$. In case of the trivial multiplier system, it is $\mathbb{Z}$.

Hence we have the following results.

Lemma 5.8. If $f_{(1)}$ and $f_{(2)}$ are Hilbert modular forms given with precision $g^{N}$, then their product $f_{(1)} f_{(2)}$ is given with precision $g^{N}$.

Lemma 5.9. (Calculation of Borcherds products with given precision.) Let $p \equiv 1(\bmod 4)$ be a prime, and for $m \in \mathbb{N}$ with $\chi_{p}(m) \geq 0$, denote by $f_{m}$ the unique basis element of $A_{0}^{+}\left(p, \chi_{p}\right)$ with Fourier expansion $s(-m)^{-1} q^{-m}+\sum_{k \geq 0} a(k) q^{k}$. Let $W$ be a Weyl chamber attached to $f_{m}$ and $\tau \in W$ with $y_{1}=\operatorname{Im}\left(\tau_{1}\right)$ and $y_{2}=\operatorname{Im}\left(\tau_{2}\right)$. Define $a(-m)=s(-m)^{-1}$ and $a(-k)=0$ for all $k \in \mathbb{N}_{0} \backslash\{m\}$. Then for every $N \in \mathbb{N}$ the Borcherds product $\Psi_{m}$, given by

$$
\begin{aligned}
\Psi_{m}\left(\tau_{1}, \tau_{2}\right)= & \mathbf{e}\left(\rho_{W} \tau_{1}+\overline{\rho_{W}} \tau_{2}\right) \\
& \times \prod_{\substack{\nu \in \mathfrak{o} / \sqrt{p} \\
(\nu, W)>0}}\left(1-\mathbf{e}\left(\nu \tau_{1}+\bar{\nu} \tau_{2}\right)\right)^{s(p \nu \bar{\nu}) a(p \nu \bar{\nu})}
\end{aligned}
$$

for all $\tau \in W$ can be calculated with precision $g^{N}$ by the following algorithm:

1: Calculate $R(W, n)$ with Lemma 4.6.

2: Calculate $\rho_{W}$ with Lemma 4.4.

3: Calculate the leading coefficient $a(h,-k) g^{-k}$ of

$$
\prod_{\nu}\left(1-\mathbf{e}\left(\nu \tau_{1}+\bar{\nu} \tau_{2}\right)\right)^{s(p \nu \bar{\nu}) a(p \nu \bar{\nu})}
$$

where the product is over

$$
\begin{aligned}
& \nu=\nu_{1}+\nu_{2} \sqrt{p} \in \mathfrak{o} / \sqrt{p}, \quad \nu_{2}^{2} \leq \nu_{1}^{2} / p+m / p^{2} \\
& -\sqrt{\left(y_{1}-y_{2}\right)^{2} m /\left(4 p y_{1} y_{2}\right)}<\nu_{1}<0
\end{aligned}
$$

4: If $\mathrm{S}\left(\rho_{W}\right)$ is negative, then rewrite $k:=k-\mathrm{S}\left(\rho_{W}\right)$.

5: Expand

$R=g^{\mathrm{S}\left(\rho_{W}\right)} h^{\left(\rho_{W}-\overline{\rho_{W}}\right) \sqrt{p}} \prod_{\nu}\left(1-g^{2 \nu_{1}} h^{2 p \nu_{2}}\right)^{s(p \nu \bar{\nu}) a(p \nu \bar{\nu})}$,

where the product over $\nu$ is as in step 3, and we expand each factor $\left(1-g^{2 \nu_{1}} h^{2 p \nu_{2}}\right)^{s(p \nu \bar{\nu}) a(p \nu \bar{\nu})}$ with precision 


\begin{tabular}{|c|c|c|c|c|c|c|}
\hline$\Psi_{k}$ & weight & divisor & $\rho_{W}$ & $\mu$ & other & diagonal \\
\hline \multicolumn{7}{|c|}{ Fourier expansion } \\
\hline \multicolumn{7}{|c|}{ Fourier expansion on the diagonal (if not 0) } \\
\hline$\Psi_{1}$ & 1 & $T_{1}$ & $\frac{1}{6}+\frac{\sqrt{13}}{26}$ & $\mu_{1,2}$ & $\overline{\Psi_{1}}=-\Psi_{1}$ & $\Psi_{1}(\tau, \tau) \equiv 0$ \\
\hline \multicolumn{7}{|c|}{$g^{1 / 3}\left(h-\frac{1}{h}\right)-g^{4 / 3}\left(2\left(h^{2}-\frac{1}{h^{2}}\right)+\left(h^{4}-\frac{1}{h^{4}}\right)\right)+O\left(g^{7 / 3}\right)$} \\
\hline$\Psi_{3}$ & 4 & $T_{3}$ & $\frac{5}{6}+5 \frac{\sqrt{13}}{26}$ & $\mu_{2,1}$ & & $\Psi(\tau, \tau)=(\eta(\tau))^{16}$ \\
\hline \multicolumn{7}{|c|}{$-g^{2 / 3}+g^{5 / 3}\left(-2\left(h+\frac{1}{h}\right)+9\left(h^{3}+\frac{1}{h^{3}}\right)+\left(h^{5}+\frac{1}{h^{5}}\right)\right)+g^{8 / 3}\left(16+O\left(h^{2}+\frac{1}{h^{2}}\right)\right)+O\left(g^{11 / 3}\right)$} \\
\hline \multicolumn{7}{|c|}{$-q^{2 / 3}+16 q^{5 / 3}-104 q^{8 / 3}+O\left(q^{11 / 3}\right)$} \\
\hline$\Psi_{4}$ & 3 & $T_{4}$ & $\frac{1}{3}+\frac{\sqrt{13}}{13}$ & $\mu_{2,1}$ & $\overline{\overline{\Psi_{4}}}=-\Psi_{4}$ & $\Psi_{4}(\tau, \tau) \equiv 0$ \\
\hline \multicolumn{7}{|c|}{$g^{2 / 3}\left(h^{2}-\frac{1}{h^{2}}\right)+g^{5 / 3}\left(-24\left(h-\frac{1}{h}\right)-16\left(h^{3}-\frac{1}{h^{3}}\right)+8\left(h^{5}-\frac{1}{h^{5}}\right)\right)+O\left(g^{8 / 3}\right)$} \\
\hline$\frac{\Psi_{4}}{2 \Psi_{1}}$ & 2 & $T_{4}-T_{1}$ & $\frac{1}{6}+\frac{\sqrt{13}}{26}$ & $\mu_{1,2}$ & symmetric & $\frac{\Psi_{4}}{2 \Psi_{1}}(\tau, \tau)=\eta^{8}(\tau)$ \\
\hline \multicolumn{7}{|c|}{$\frac{1}{2} g^{1 / 3}\left(h+\frac{1}{h}\right)+g^{4 / 3}\left(-26-4\left(h^{2}+\frac{1}{h^{2}}\right)+9\left(h^{4}+\frac{1}{h^{4}}\right)\right)+O\left(g^{7 / 3}\right)$} \\
\hline \multicolumn{7}{|c|}{$-q^{1 / 3}\left(-1+8 q-20 q^{2}+70 q^{4}\right)+O\left(q^{16 / 3}\right)$} \\
\hline$\Psi_{10}$ & 4 & $T_{10}$ & 0 & 1 & $\overline{\overline{\Psi_{10}}}=\Psi_{10}$ & $\Psi_{10}(\tau, \tau)=\left(E_{4}(\tau)\right)^{2}$ \\
\hline \multicolumn{7}{|c|}{$1+g\left(200\left(h+\frac{1}{h}\right)+40\left(h^{3}+\frac{1}{h^{3}}\right)\right)+O\left(g^{2}\right)$} \\
\hline \multicolumn{7}{|c|}{$1+480 q+61920 q^{2}+1050240 q^{3}+7926240 q^{4}+O\left(q^{5}\right)$} \\
\hline$\Psi_{13}$ & 7 & $T_{13}$ & $\frac{1}{3}$ & $\mu_{2,1}$ & symmetric & $\Psi_{13}(\tau, \tau)=\eta^{16}(\tau) \cdot E_{6}(\tau)$ \\
\hline \multicolumn{7}{|c|}{$g^{2 / 3}+g^{5 / 3}\left(-221\left(h+\frac{1}{h}\right)-39\left(h^{3}+\frac{1}{h^{3}}\right)\right)$} \\
\hline \multicolumn{7}{|c|}{$q^{2 / 3}-520 q^{5 / 3}-8464 q^{8 / 3}+O\left(q^{11 / 3}\right)$} \\
\hline$\Psi_{14}$ & 6 & $T_{14}$ & 0 & 1 & $\overline{\Psi_{14}}=\Psi_{14}$ & $\Psi_{14}(\tau, \tau)=E_{6}^{2}(\tau)$ \\
\hline \multicolumn{7}{|c|}{$1-504 g\left(h+\frac{1}{h}\right)+O\left(g^{2}\right)$} \\
\hline \multicolumn{7}{|c|}{$1-1008 q+220752 q^{2}+16519104 q^{3}+399517776 q^{4}+O\left(q^{5}\right)$} \\
\hline \multicolumn{7}{|c|}{ The restriction of $\Psi_{14}$ to the diagonal has trivial character. } \\
\hline$\Psi_{26}$ & 6 & $T_{26}$ & 0 & 1 & $\overline{\Psi_{26}}=\Psi_{26}$ & $\Psi_{26}(\tau, \tau)=\frac{125}{27}\left(E_{6}(\tau)\right)^{2}-\frac{98}{27}\left(E_{4}(\tau)\right)^{3}$ \\
\hline \multicolumn{7}{|c|}{$1-g\left(3432\left(h+\frac{1}{h}\right)+208\left(h^{3}+\frac{1}{h^{3}}\right)\right)+O\left(g^{2}\right)$} \\
\hline & & $1-72$ & $0 q+37128$ & $q^{2}+1$ & $4938560 q^{3}+$ & $408750160 q^{4}+O\left(q^{5}\right)$ \\
\hline
\end{tabular}

TABLE 11. Borcherds products in case $p=13$ for the Weyl chamber $W\left(-i \overline{\varepsilon_{0}}, i \varepsilon_{0}\right)\left(g=\exp \left(\pi i\left(\tau_{1}+\tau_{2}\right)\right)\right.$ and $h=$ $\left.\exp \left(\pi i\left(\tau_{1}-\tau_{2}\right) / \sqrt{p}\right)\right)$.

$g^{k+N}$ and neglect higher-order terms. For negative exponents use the geometric series

$$
(1-x)^{-1}=\sum_{n=0}^{\infty} x^{n} \quad \text { for }|x|<1 .
$$

Then $\Psi_{m}$ is given by $R$ with precision $g^{N}$.

Proof. Let $\mu=\mu_{1}+\mu_{2} \sqrt{p} \in \mathfrak{o} / \sqrt{p}$. Then $\mathbf{e}(\mathrm{S}(\nu \tau))=$ $g^{2 \nu_{1}} h^{2 p \nu_{2}}$ and the factor $\left(1-g^{2 \nu_{1}} h^{2 p \nu_{2}}\right)$ has a negative power of $g$ if and only if $\nu_{1}<0$. In this case we get from
$(W, \nu)>0$ the following:

$$
\begin{aligned}
& \nu_{1}\left(y_{1}+y_{2}\right)+\nu_{2}\left(y_{1}-y_{2}\right) \sqrt{p}>0 \\
& \Longleftrightarrow \nu_{2}\left(y_{1}-y_{2}\right) \sqrt{p}>\underbrace{-\nu_{1}}_{>0} \underbrace{\left(y_{1}+y_{2}\right)}_{>0} \\
& \Longleftrightarrow\left|\nu_{2}\right|\left|y_{1}-y_{2}\right| \sqrt{p}>-\nu_{1}\left(y_{1}+y_{2}\right) \\
& \Longleftrightarrow\left|\nu_{2}\right|>\left|\nu_{1}\right| \frac{y_{1}+y_{2}}{\left|y_{1}-y_{2}\right| \sqrt{p}} .
\end{aligned}
$$




\begin{tabular}{|c|c|c|c|c|c|c|}
\hline$\Psi_{k}$ & weight & divisor & $\rho_{W}$ & $\mu$ & other & diagonal \\
\hline \multicolumn{7}{|c|}{ Fourier expansion } \\
\hline \multicolumn{7}{|c|}{ Fourier expansion on the diagonal (if not 0 ) } \\
\hline$\Psi_{1}$ & $\frac{1}{2}$ & $T_{1}$ & $\frac{1}{8}+\frac{\sqrt{17}}{34}$ & $\mu_{3,4}$ & $\overline{\Psi_{1}}=-\Psi_{1}$ & 0 \\
\hline \multicolumn{7}{|c|}{$g^{1 / 4}\left(h-h^{-1}\right)-g^{5 / 4}\left(\left(h^{2}-\frac{1}{h^{2}}\right)+\left(h^{4}-\frac{1}{h^{4}}\right)\right)+g^{9 / 4}\left(h^{9}-\frac{1}{h^{9}}\right)+O\left(g^{13 / 4}\right)$} \\
\hline$\Psi_{2}$ & $\frac{3}{2}$ & $T_{2}$ & $\frac{5}{8}+\frac{5 \sqrt{17}}{34}$ & $\mu_{3,3}=\mu_{3,4}^{5}$ & $\overline{\overline{\Psi_{2}}}=\Psi_{2}$ & $\Psi_{2}(\tau, \tau)=-(\eta(\tau))^{6}$ \\
\hline \multicolumn{7}{|c|}{$-g^{1 / 4}+g^{5 / 4}\left(-\left(h+\frac{1}{h}\right)+3\left(h^{3}+\frac{1}{h^{3}}\right)+\left(h^{5}+\frac{1}{h^{5}}\right)\right)+O\left(g^{9 / 4}\right)$} \\
\hline \multicolumn{7}{|c|}{$-q^{1 / 4}+6 q^{5 / 4}-9 q^{9 / 4}-10 q^{13 / 4}+30 q^{17 / 4}+O\left(q^{21 / 4}\right)$} \\
\hline$\Psi_{4}$ & $\frac{7}{2}$ & $T_{4}$ & $\frac{15}{8}+\frac{15 \sqrt{17}}{34}$ & $\mu_{3,5}=\mu_{3,4}^{7}$ & $\overline{\Psi_{4}}=-\Psi_{4}$ & 0 \\
\hline \multicolumn{7}{|c|}{$-g^{3 / 4}\left(h^{2}-\frac{1}{h^{2}}\right)+g^{7 / 4}\left(13\left(h+\frac{1}{h}\right)+11\left(h^{3}-\frac{1}{h^{3}}\right)-2\left(h^{5}-\frac{1}{h^{5}}\right)\right)+O\left(g^{11 / 4}\right)$} \\
\hline$\frac{\Psi_{4}}{\Psi 1}$ & 3 & $T_{4}-T_{1}$ & $\frac{7}{4}+\frac{7 \sqrt{17}}{17}$ & $\mu_{2,3}=\mu_{3,4}^{6}$ & $\overline{\frac{\Psi_{4}}{\Psi_{1}}}=\frac{\Psi_{4}}{\Psi_{1}}$ & $\frac{\Psi_{4}}{\Psi_{1}}=2(\eta(\tau))^{12}$ \\
\hline$\Psi_{8}$ & $\frac{15}{2}$ & $T_{8}$ & $\frac{17}{8}+\frac{\sqrt{17}}{2}$ & $\mu_{3,4}$ & $\overline{\Psi_{8}}=\Psi_{8}$ & $\Psi_{8}(\tau, \tau)=(\eta(\tau))^{30}=\Delta(\tau) \cdot(\eta(\tau))^{6}$ \\
\hline \multicolumn{7}{|c|}{$g^{5 / 4}+g^{9 / 4}\left(10\left(h+\frac{1}{h}\right)-24\left(h^{3}+\frac{1}{h^{3}}\right)-\left(h^{7}+\frac{1}{h^{7}}\right)\right)+O\left(g^{13 / 4}\right)$} \\
\hline$\Psi_{9}$ & $\frac{7}{2}$ & $T_{9}$ & $\frac{3}{8}+\frac{3 \sqrt{17}}{34}$ & $\mu_{3,6}=\mu_{3,4}^{3}$ & $\overline{\Psi_{9}}=-\Psi_{9}$ & 0 \\
\hline \multicolumn{7}{|c|}{$g^{3 / 4}\left(h^{3}-h^{-3}\right)+g^{7 / 4}\left(-36\left(h^{2}-\frac{1}{h^{2}}\right)-36\left(h^{4}-\frac{1}{h^{4}}\right)+27\left(h^{6}-\frac{1}{h^{6}}\right)\right)+O\left(g^{7 / 4}\right)$} \\
\hline$\frac{\Psi_{9}}{\Psi_{1}}$ & 3 & $T_{9}-T_{1}$ & $\frac{1}{4}+\frac{\sqrt{17}}{17}$ & $\mu_{2,2}=\mu_{3,4}^{2}$ & $\overline{\frac{\Psi_{9}}{\Psi_{1}}}=\frac{\Psi_{9}}{\Psi_{1}}$ & $\frac{\Psi_{9}}{\Psi_{1}}(\tau, \tau)=3 \cdot(\eta(\tau))^{12}$ \\
\hline \multicolumn{7}{|c|}{$g^{1 / 2}\left(h^{2}+1+\frac{1}{h^{2}}\right) g^{3 / 2}\left(-40\left(h+\frac{1}{h}\right)-6\left(h^{3}+\frac{1}{h^{3}}\right)+28\left(h^{5}+\frac{1}{h^{5}}\right)\right)+O\left(g^{5 / 2}\right)$} \\
\hline$\Psi_{13}$ & 7 & $T_{13}$ & $\frac{9}{4}+\frac{9 \sqrt{17}}{17}$ & $\mu_{2,2}=\mu_{3,4}^{2}$ & $\overline{\Psi_{13}}=\Psi_{13}$ & $\Psi_{13}(\tau, \tau)=-E_{4}(\tau)^{2} \cdot(\eta(\tau))^{12}$ \\
\hline$\Psi_{15}$ & 4 & $T_{15}$ & 0 & 1 & $\overline{\Psi_{15}}=\Psi_{15}$ & $\Psi_{15}(\tau, \tau)=E_{4}^{2}(\tau)=E_{8}(\tau)$ \\
\hline \multicolumn{7}{|c|}{$1+240 g\left(h+\frac{1}{h}\right)+O\left(g^{2}\right)$} \\
\hline \multicolumn{7}{|c|}{$1+480 q+61920 q^{2}+1050240 q^{3}+7926240 q^{4}+O\left(q^{5}\right)$} \\
\hline$\Psi_{17}$ & $\frac{9}{2}$ & $T_{17}$ & $\frac{1}{8}$ & $\mu_{3,3}=\mu_{3,4}^{5}$ & $\overline{\Psi_{17}}=\Psi_{17}$ & $\Psi_{17}(\tau, \tau)=(\eta(\tau))^{6} \cdot E_{6}(\tau)$ \\
\hline \multicolumn{7}{|c|}{$g^{1 / 4}-g^{5 / 4}\left(204\left(h+\frac{1}{h}\right)+51\left(h^{3}+\frac{1}{h^{3}}\right)\right)+O\left(g^{9 / 4}\right)$} \\
\hline \multicolumn{7}{|c|}{$q^{1 / 4}-510 q^{5 / 4}-13599 q^{9 / 4}-27710 q^{13 / 4}+50370 q^{17 / 4}+360194 q^{21 / 4}-19479432 q^{25 / 4}$} \\
\hline$\Psi_{21}$ & 6 & $T_{21}$ & 0 & 1 & $\overline{\Psi_{21}}=\Psi_{21}$ & $\Psi_{21}=E_{6}^{2}(\tau)$ \\
\hline \multicolumn{7}{|c|}{$1-630 g\left(h+h^{-1}\right)+126 g\left(h^{3}+h^{-3}\right)+O\left(g^{2}\right)$} \\
\hline \multicolumn{7}{|c|}{$1-1008 q+220752 q^{2}+16519104 q^{3}+399517776 q^{4}+O\left(q^{5}\right)$} \\
\hline
\end{tabular}

TABLE 12. Borcherds products in case $p=17$ for the Weyl chamber $W\left(-i \overline{\varepsilon_{0}}, i \varepsilon_{0}\right)\left(g=\exp \left(\pi i\left(\tau_{1}+\tau_{2}\right)\right)\right.$ and $h=$ $\left.\exp \left(\pi i\left(\tau_{1}-\tau_{2}\right) / \sqrt{p}\right)\right)$.

Moreover, for $\mathrm{N}(\nu)<-m / p$ we have $a(p \mathrm{~N}(\nu))=0$, so we can skip

$$
\left(1-\mathbf{e}\left(\nu_{1} \tau_{1}+\nu_{2} \tau_{2}\right)\right)^{s(p \mathrm{~N}(\nu)) a(p \mathrm{~N}(\nu))}=1
$$

in the product expansion of $\Psi_{m}$ whenever $\mathrm{N}(\nu)<-m / p$. So negative exponents may derive only from the factor $\mathbf{e}\left(\rho_{W} \tau_{1}+\overline{\rho_{W}} \tau_{2}\right)$ and $\nu \in \mathfrak{o} / \sqrt{p}$ with $(W, \nu)>0$ and

$$
\mathrm{N}(\nu)=\nu_{1}^{2}-p \nu_{2}^{2} \geq-m / p \quad \Longleftrightarrow \quad \nu_{2}^{2} \leq \frac{\nu_{1}^{2}}{p}+\frac{m}{p^{2}} .
$$

The combination of both conditions gives

$$
\begin{aligned}
\left|\nu_{1}\right|^{2} & \frac{\left(y_{1}+y_{2}\right)^{2}}{\left|y_{1}-y_{2}\right|^{2} p}<\nu_{2}^{2} \leq \frac{\nu_{1}^{2}}{p}+\frac{m}{p^{2}} \\
& \Longrightarrow \frac{\left|\nu_{1}\right|^{2}}{p}\left(\frac{\left(y_{1}+y_{2}\right)^{2}}{\left|y_{1}-y_{2}\right|^{2}}-1\right)<\frac{m}{p^{2}} \\
& \Longrightarrow\left|\nu_{1}\right|^{2} \frac{\left(y_{1}+y_{2}\right)^{2}-\left(y_{1}-y_{2}\right)^{2}}{\left(y_{1}-y_{2}\right)^{2}}<\frac{m}{p} \\
& \Longrightarrow\left|\nu_{1}\right|^{2}<\frac{m}{p} \frac{\left(y_{1}-y_{2}\right)^{2}}{4 y_{1} y_{2}} .
\end{aligned}
$$


Since $s(-m) a(-m)=1$, every factor $\left(1-q^{2 \nu_{1}} h^{2 p \nu_{2}}\right)$ with negative $q$-exponent occurs once, so by Lemma 5.8 , we need every factor in the product expansion of $\Psi_{m}$ with precision $g^{N+k}$. It remains to show that the geometric series can be applied for negative exponents. Since $\nu \operatorname{Im}\left(\tau_{1}\right)+\bar{\nu} \operatorname{Im}\left(\tau_{2}\right)>0$, by $(W, \nu)>0$ and

$$
\left|\mathbf{e}\left(\nu \tau_{1}+\bar{\nu} \tau_{2}\right)\right|=e^{-2 \pi \nu \operatorname{Im}\left(\tau_{1}\right)+\bar{\nu} \operatorname{Im}\left(\tau_{2}\right)}<1,
$$

the geometric series converges.

Remark 5.10. Some results of these calculations can be found in Tables 10, 11, and 12. The full data and the corresponding Maple worksheets can be found at http://www.matha.rwth-aachen.de.

\section{REFERENCES}

[Borcherds 98] R. E. Borcherds. "Automorphic Forms with Singularities on Grassmannians." Inventiones mathematicae 132 (1998), 491-562.
[Brunier and Bundschuh 03] J. H. Bruinier and M. Bundschuh. "On Borcherds Products Associated with Lattices of Prime Discriminant." Ramanujan Journal 7 (2003), 49-61.

[van der Geer 88] G. van der Geer. Hilbert Modular Surfaces, Ergebnisse der Mathematik und ihrer Grenzgebiete 16. New York: Springer, 1988.

[Gundlach 88] K.-B. Gundlach. "Die Berechnung von Multiplikatorsystemen zu Hilbertschen Modulgruppen." Nachrichten der Akademie der Wissenschaften Göttingen, II. Mathematisch-Physikalische Klasse 7 (1988), 1-28.

[Mayer 07] S. Mayer. "Hilbert Modular Forms for the Fields $\mathbb{Q}(\sqrt{5}), \mathbb{Q}(\sqrt{13})$ and $\mathbb{Q}(\sqrt{17}) . " \quad \mathrm{PhD}$ thesis, Aachen, 2007. Available online (http://darwin.bth.rwth-aachen.de/ opus3/volltexte/2007/1985/).

[Mayer 09] S. Mayer. "Rings of Hilbert Modular Forms for the Fields $\mathbb{Q}(\sqrt{13})$ and $\mathbb{Q}(\sqrt{17})$." To appear, 2009.

[Siegel 69] C. L. Siegel. "Berechnung von Zetafunktionen an ganzzahligen Stellen." Nachrichten der Akademie der Wissenschaften Göttingen, II. Mathematisch-Physikalische Klasse 10 (1969), 87-102.

Sebastian Mayer, Lehrstuhl A für Mathematik, RWTH Aachen University, 52056 Aachen, Germany

(Sebastian.Mayer@matha.rwth-aachen.de)

Received December 16, 2008; accepted April 17, 2009. 